Draft Version September 4, 2018

Preprint typeset using $\mathrm{L}^{A} \mathrm{~T}_{\mathrm{E}} \mathrm{X}$ style emulateapj v. 04/20/08

\title{
TWIN BINARIES: STUDIES OF STABILITY, MASS TRANSFER, AND COALESCENCE
}

\author{
J. C. Lombardi, Jr. ${ }^{1}$, W. Holtzman ${ }^{1}$, K. L. Dooley ${ }^{2}$, K. Gearity ${ }^{1}$, V. Kalogera ${ }^{3,4}$, F. A. Rasio ${ }^{3,4}$ \\ Draft version September 4, 2018
}

\begin{abstract}
Motivated by suggestions that binaries with almost equal-mass components ("twins") play an important role in the formation of double neutron stars and may be rather abundant among binaries, we study the stability of synchronized close and contact binaries with identical components in circular orbits. In particular, we investigate the dependency of the innermost stable circular orbit on the core mass, and we study the coalescence of the binary that occurs at smaller separations. For twin binaries composed of convective main-sequence stars, subgiants, or giants with low mass cores $\left(M_{c} \lesssim 0.15 M\right.$, where $M$ is the mass of a component), a secular instability is reached during the contact phase, accompanied by a dynamical mass transfer instability at the same or at a slightly smaller orbital separation. Binaries that come inside this instability limit transfer mass gradually from one component to the other and then coalesce quickly as mass is lost through the outer Lagrangian points. For twin giant binaries with moderate to massive cores $\left(M_{c} \gtrsim 0.15 M\right)$, we find that stable contact configurations exist at all separations down to the Roche limit, when mass shedding through the outer Lagrangian points triggers a coalescence of the envelopes and leaves the cores orbiting in a central tight binary. In addition to the formation of binary neutron stars, we also discuss the implications of our results for the production of planetary nebulae with double degenerate central binaries.

Subject headings: binaries: close — binaries: general - hydrodynamics — instabilities - methods: numerical - stars: general
\end{abstract}

\section{INTRODUCTION AND MOTIVATION}

\subsection{Formation of Binary Neutron Stars}

The evolutionary history and formation of close binaries with two neutron stars similar to the Hulse-Taylor pulsar B1913+16 (Hulse \& Taylor 1975) and the double pulsar J0737-3039 (Burgay et al. 2003) is a topic of intense current interest. Most recent studies of the known double neutron stars focus on the stages going back to the time of the second supernova explosion and the formation of the youngest of the two neutron stars (Dewi \& van den Heuvel 2004; Willems \& Kalogera 2004; Willems et al. 2004; Piran \& Shaviv 2005; Stairs et al. 2006; Wang, Lai, \& Han 2006; Wong et al. 2010, and references therein). Although these studies provide very interesting constraints on the properties of the stellar progenitor of the second neutron star, they do not probe the earlier evolutionary history. That part remains uncertain and more difficult to constrain empirically based on the measured properties of observed systems.

Since the discovery of the Hulse-Taylor binary, the origin of double neutron stars has been naturally connected to the evolution of massive binaries, with stellar components that are massive enough to form two neutron stars at the end of their lifetime. Over the years, a qualitative consensus of understanding for the formation of double neutron stars developed (see, e.g., Bhattacharya \& van den Heuvel 1991): massive binaries experience a

\footnotetext{
${ }^{1}$ Department of Physics, Allegheny College, Meadville, PA, 16335

${ }^{2}$ Department of Physics, University of Florida, Gainesville, FL 32611

3 Department of Physics and Astronomy, Northwestern University, 2145 Sheridan Rd., Evanston, IL 60208

${ }_{4}^{4}$ Center for Interdisciplinary Exploration and Research in Astrophysics (CIERA), Northwestern University.
}

phase of stable mass transfer when the primary overflows its Roche lobe revealing its helium core; this core ends its lifetime in a supernova forming the first neutron star in the system; the binary becomes a high-mass $\mathrm{X}$-ray binary until the massive secondary fills its Roche lobe and the binary enters a dynamically unstable phase of mass transfer leading to inspiral in a common envelope that engulfs the neutron star; during this phase the neutron star is thought to be spun up through recycling and, if the binary avoids a merger in the inspiral, the helium core of the secondary is revealed after the envelope ejection; this core explodes in a supernova, forming the second neutron star in the system, and the double neutron star further evolves through orbital contraction and gravitational-wave emission. Variations of this evolutionary sequence have been shown to be realized by theoretical binary population studies (e.g., Belczynski et al. 2002).

Brown (1995) argued that the inspiral of the neutron star during the common envelope phase in the standard model is problematic: the neutron star is expected to experience hypercritical accretion (Chevalier 1993) at rates many orders of magnitude above the photon Eddington limit through a neutrino-cooled accretion flow (see also Fryer et al. 1996). Such a rapid accretion phase along with the adoption of a low maximum neutron star mass $\left(\sim 1.5 \mathrm{M}_{\odot}\right.$ derived for a soft equation of state for neutron star matter with kaon condensation) led Brown to conclude that all neutron stars in common envelope phases will accrete enough matter to collapse into a black hole. Consequently, he argued that the "standard" evolutionary sequence described above aborts the formation of double neutron stars and instead leads to the formation of binaries with low-mass black holes and neutron star companions. We note however that, even with the same 
treatment of hypercritical accretion, a maximum neutron star mass of $\sim 2 M_{\odot}$ (corresponding to a more regular stiff equation of state) does prevent a good fraction of neutron stars from being transformed into low-mass black holes (Belczynski et al. 2002).

Brown (1995) also noted how the masses measured in known double neutron stars are very close to being equal (Nice et al. 1996; Thorsett \& Chakrabarty 1999; Stairs et al. 2002; Weisberg \& Taylor 2005; Jacoby et al. 2006; Kramer et al. 2006, and references therein). Motivated by these two points, he proposed a different formation channel for double neutron stars. Brown suggested that double neutron stars form from massive binaries with component masses that are within $\sim 4 \%$ of one another. Consequently the red giant phases of the two components coincide in time and when mass transfer ensues from the primary, both components have deep convective envelopes and well developed helium cores, so that a double core phase develops, where the two helium cores orbit within the combined envelopes of the two massive stars. Provided that there is enough orbital energy, the common envelope is ejected before the two cores merge, and a tight binary with two helium cores is formed. These two cores differ very little in mass and reach core collapse one very soon after the other $\left(\sim 10^{3} \mathrm{yr}\right.$ based on helium-star models, Habets 1986; Pols 1994), forming a close double neutron star.

The advantages of this hypothesized evolutionary channel are (i) a neutron star never experiences common envelope spiral-in and hypercritical accretion, and (ii) the two stellar components have so similar masses that they naturally form neutron stars of almost equal mass as observed (Bethe \& Brown 1998; Bethe, Brown, \& Lee 2007). On the other hand, this channel (i) requires that mass transfer between the red giant progenitors will indeed lead to the inspiral of the two cores in a common envelope and (ii) requires fine-tuning the conditions for recycling the first neutron star, as this spin up must occur during the very short interval between the two supernovae through the stellar wind or possibly during the brief Roche-lobe overflow from the lower-mass helium star (Dewi et al. 2006). A potential additional disadvantage is that this channel is very restrictive in that it requires progenitors that are at most only $\sim 4 \%$ apart in mass: however, in their study of protobinary stars, Krumholz \& Thompson (2007) find that for a wide range of initial conditions Roche lobe overflow tends to equalize the masses of the binary components.

The double neutron star formation channel suggested by Brown (1995) has attracted renewed attention because of the reported abundance of "twins," massive binaries with mass ratios very close to unity (within 5\%) by Pinsonneault \& Stanek (2006). Specifically they analyze data for 21 detached eclipsing binaries in the Small Magellanic Cloud and find that the data are consistent with a flat mass function containing $55 \%$ of the systems and "twins" with mass ratios greater than 0.95 containing the remaining $45 \%$ of the population. However, it is important to note that there are severe selection effects against the discovery of binaries with small mass ratios (typically $\lesssim 0.5$, Hogeveen $1992 \mathrm{a}, \mathrm{b}, \mathrm{c}$ ); therefore the contribution of twins may not be as significant as implied by the most recent observations. Quantitative modeling of the associated selection effects is required to derive more reliable statistical conclusions.

Apart from uncertainties with the initial properties of the binary population, a number of physical processes related to these formation channels make it hard to assess their relative contribution to double neutron star formation. The physics of common envelopes and hypercritical accretion, as well as of neutron star equations of state and their maximum mass, is not well understood (but see Lee, Park, \& Brown 2007). Also, the development of a dynamical instability and subsequent common envelope phase with the inspiral of the two cores is assumed, but has not been investigated before in any detail. In this study, we attempt to understand better one of the aspects related to the formation channel suggested by Brown (1995): the fate of mass transfer between two stars of almost equal mass.

\subsection{Binaries and Planetary Nebulae}

Although our primary motivation is to investigate a formation channel for binary neutron stars, our results are relevant to other scenarios as well. For example, through common envelope evolution, binaries could transform quite naturally into planetary nebulae (PNe) with a central close binary. The influence of binaries on the production and morphology of PNe has received increased attention in recent years (e.g., Miszalski et al. 2009; Jones et al. 2010), and observations to date have identified approximately 40 close binaries in the centers of PNe: see De Marco (2009) for a summary of both the relevant theory and observations. As lifetimes of $\mathrm{PNe}$ are less than only $10^{5}$ years, an embedded central binary has undergone no significant evolution since the common envelope phase that presumably formed it.

About a quarter of the known close binary systems within $\mathrm{PNe}$ are thought to be double degenerates, that is, binaries in which the components are pre-white dwarfs (also known as extreme horizontal branch stars, hot subdwarfs, or subdwarf B and O stars) or white dwarfs. Such double degenerate binaries, with or without planetary nebulae, are particularly interesting. As possible progenitors for Type Ia supernovae, they are the desired targets of the ESO SN Ia Progenitor Survey (see Geier et al. 2011, and references therein).

Interestingly, three of the five well studied double degenerate binaries within PNe (see Hillwig 2011, and references therein) may contain nearly equal mass components and therefore are possibly the progeny of twin binaries. In particular, models of a double hot subdwarf binary with $q \approx 1$ are consistent with photometric and spectroscopic observations of the central stars in NGC 6026 (Hillwig et al. 2010) and in Abell 41 (Bruch et al. 2001; Shimanskii et al. 2008). Similarly, recent observations of the central star in Hen 2-428 reveal it also to be a double degenerate binary, with the effective temperature of the components being within a few thousand Kelvin (Santander-Garcia et al. 2011), suggesting a mass ratio near $q=1$. As we investigate in this paper, the coalescence of a twin giant binary could lead to the formation of double degenerate core surrounded by a circumbinary envelope, a type of proto-PN.

\subsection{Theoretical Work on Close Binaries}

Most of the classical theoretical work on close binaries was done in the limit of a self-gravitating incom- 
pressible fluid (see Chandrasekhar 1969, and references therein). An essential result found in the incompressible case is that the hydrostatic equilibrium solutions for sufficiently close binaries can become globally unstable (Chandrasekhar 1975; Tassoul 1975). The classical analytic studies for binaries were extended to polytropes in the work of Lai et al. (1993a,b, 1994a,b,c). In their approach, the stars are modeled as self-gravitating compressible ellipsoids, and an energy variational principle is used to construct approximate equilibrium configurations and study their stability. These treatments, along with complementary numerical hydrodynamic calculations (Rasio \& Shapiro 1992, 1994, 1995), demonstrate that dynamical instabilities persist in the compressible regime and can cause a binary to coalesce to form a rapidly rotating spheroidal object. Such a dynamical instability can trigger a merger in just a few orbital periods (Rasio \& Shapiro 1992) or an episode of mass transfer that lasts many orbits (Motl et al. 2002; D'Souza et al. 2006; Frank 2008; Dan et al. 2009; Lorén-Aguilar et al. 2009).

The evolution of a close binary system can also be affected by another type of global instability. It has been referred to by various names, such as the secular instability (Lai et al. 1993a,b, 1994a,b,c), tidal instability (Counselman 1973; Hut 1980), gravogyro instability (Hachisu \& Eriguchi 1984), and Darwin instability (Levine et al. 1993). Its physical origin is easy to understand (Lai et al. 1993a, 1994b; Rasio 1994; Webbink 2006). There exists a minimum value of the total angular momentum $J$ for a synchronized close binary. This is simply because the spin angular momentum, which increases as the separation $r$ decreases for a synchronized system, can become comparable to the orbital angular momentum for sufficiently small $r$. A system that reaches the minimum of $J$ cannot evolve further by angular momentum loss and remain synchronized. Instead, the combined action of tidal forces and viscous dissipation will drive the system out of synchronization and cause rapid orbital decay as angular momentum is continually transferred from the orbit to the spins. The orbital decay then proceeds on a timescale comparable to the synchronization time of a stable binary.

In this paper, we pay particular attention to the onset of orbital instabilities, including those characterized by mass transfer, as well as the subsequent inspiral of the cores. We extend previous hydrodynamic studies of close binary systems to cases that involve identical giants, that is, to twin stars with dense stellar cores and extended envelopes. So that our results can be scaled to stars of any size or mass, we approximate each star as a condensed polytrope, namely, as a point mass surrounded by a uniform specific entropy fluid of adiabatic index $\Gamma=5 / 3$. Such a model is appropriate for a fully convective monatomic ideal gas surrounding a compact core. We consider fractional core masses $m_{c}$ that cover the entire range of theoretical possibilities. Zero core mass models correspond to normal (or "complete") $n=1.5$ polytropes, which are most appropriate for low mass main-sequence stars and non-relativistic white dwarfs. At the other extreme, when $m_{c}=1$, there is no mass in the gaseous envelope, and the binary is simply that of two point masses. By varying the core mass between these extremes, we are able to study in a systematic way the full parameter space of twin binaries and determine under what conditions mass transfer develops or an innermost stable circular orbit exists.

For typical compositions, the subgiant phase begins when the core mass grows to $\sim 10 \%$ of the total mass, the so-called Schönberg-Chandrasekhar limit (Schönberg $\&$ Chandrasekhar 1942). By the time the star reaches the base of the red giant branch, the core mass has increased by a few more percent of the total mass. Roughly speaking then, our models with $m_{c} \lesssim 0.1,0.1 \lesssim m_{c} \lesssim 0.13$, and $m_{c} \gtrsim 0.13$ correspond to main sequence, subgiant, and giant stars, respectively. The assumption that the stellar envelope has constant specific entropy makes our models most relevant to stars that are fully convective (as with low mass main sequence stars) or that have deep convective envelopes (as in many red giants).

The use of condensed polytropes as a model of red giants has a rich history, including seminal work by Chandrasekhar (1939), Osterbrock (1953), and Härm \& Schwarzschild (1955). Mass transfer in close binary systems has been modeled with the help of condensed polytropes as well: the response of the donor due to mass loss is considered, with its resulting contraction, or expansion, compared against that of its Roche lobe (Paczyiński \& Sienkiewicz 1972; Hjellming \& Webbink 1987). If at the onset of mass transfer the star contracts less rapidly than its Roche lobe (or expands more rapidly than it), then the mass exchange is dynamically unstable, and the binary will evolve rapidly toward a new, often qualitatively different, equilibrium. Hjellming \& Webbink (1987) find that equal mass condensed polytrope contact binaries with fractional core masses $m_{c} \lesssim 0.46 \mathrm{ex}-$ perience stable mass transfer. More recently, Krumholz \& Thompson (2007) have used condensed polytropes to study the formation of twin star systems.

Although essential for a qualitative understanding of mass transfer, such treatments of close binaries do, however, make several simplifying approximations: most importantly, (i) the dynamics of the orbit and size of the Roche lobe are treated in the point mass approximation, (ii) the response of the binary components to mass loss or gain is modeled as if each star were spherical and in isolation, and (iii) mass that overflows a Roche lobe is considered to leave that star. These approximations are quite reasonable for semidetached binaries, but their validity can be questioned for contact binaries. In such cases, a common envelope persists in equilibrium outside of the Roche lobes (the inner Lagrangian surface) so that the pressure and density on the Roche lobes are non-zero: mass that overflows a Roche lobe is not necessarily transferred to the other star but rather can persist in equilibrium inside the outer Lagrangian surface. A primary goal of this paper is therefore to relax the approximations of previous works by using accurate hydrodynamical calculations to study contact binary systems.

Our paper is organized as follows. In $\S 2$ we review our numerical method and general conventions. In $\S 3$ we present our results for the equilibrium and stability properties of twin binary systems. The dynamical evolution to complete coalescence is followed for several unstable systems. Implications of our results are discussed in $\S 4$.

\section{NUMERICAL METHODS AND ASSUMPTIONS}




\subsection{The SPH code}

To generate our models, we use a modified version of the SPH code originally developed by Rasio (1991) that has been updated to include the variational equations of motion derived in Gaburov et al. (2010). SPH is a Lagrangian particle method, meaning that the fluid is represented by a finite number of fluid elements or "particles." Associated with each particle $i$ are, for example, its position $\mathbf{r}_{i}$, velocity $\mathbf{v}_{i}$, and mass $m_{i}$. Each particle also carries a purely numerical smoothing length $h_{i}$ that determines the local spatial resolution and is used in the calculation of fluid properties such as acceleration and density. Details of our SPH code, such as the particular form of the artificial viscosity $\Pi_{i j}$ and smoothing kernel $W_{i j}$ implemented, are described in Gaburov et al. (2010). See Rasio \& Lombardi (1999) and Rosswog (2009) for reviews of SPH.

Because the gas in our initial stellar models is of constant specific entropy, we find it convenient to integrate the so-called entropic variable $A_{i}$ of each particle $i$. The entropic variable is simply the proportionality constant in the polytropic equation of state $p=A \rho^{\Gamma}$, where $p$ is pressure and $\rho$ is density. The entropic variable is so named because of its close connection to entropy: both quantities are conserved in reversible processes and strictly increase otherwise. We therefore use $d A_{i} / d t=0$ in the relaxations of our single star models and in the calculations of our binary equilibrium sequences. For our dynamical calculations of merger scenarios (see $\S 2.4$ and $\S 3.2$ ), we evolve $A_{i}$ according to the discretized SPH version of the first law of thermodynamics:

$$
\frac{d A_{i}}{d t}=\frac{\Gamma-1}{2 \rho_{i}^{\Gamma-1}} \sum_{j} m_{j} \Pi_{i j}\left(\mathbf{v}_{i}-\mathbf{v}_{j}\right) \cdot \nabla_{i} W_{i j}\left(h_{i}\right) .
$$

To calculate the gravitational accelerations and potentials, we use direct summation on NVIDIA graphics cards, softening with the usual SPH kernel as in Hernquist \& Katz (1989). The use of such a softening with finite extent (as opposed, for example, to Plummer softening) increases the accuracy and stability of our SPH models, consistent with the studies of Athanassoula et al. (2000) and Dehnen (2001). The gravity of core points in our models is similarly softened, applying a constant smoothing length comparable to the minimum smoothing length in the system.

\subsection{Single Star Models}

In this section we present our procedure for modeling the stars that are used in the binary simulations of $\S 3$. Hydrodynamically, a subgiant or giant can be treated as a two-component system: a high-density, degenerate core, surrounded by an extended envelope. The very large density contrast between the core and the envelope, along with the small core radius, justifies the use of a single point mass to represent the core. Because the giants we wish to model mostly have deep convective envelopes with an equation of state dominated by monatomic ideal gas pressure, we treat their gas as a constant specific entropy fluid with an adiabatic index $\Gamma=5 / 3$.

Specifically, our stellar models are the so-called condensed polytropes, namely, constant specific entropy fluid surrounding a point mass core (Chandrasekhar 1939; Härm \& Schwarzschild 1955), which we parameterize by the core mass $m_{c}$. Each of the condensed polytropes in our family of models has total mass $M=1$ and radius $R=1$. The unit system is completed by choosing Newton's gravitational constant $G=1$. Condensed polytrope models have not only the advantage of reproducibility but also of scalability to any stellar mass and radius: although our focus here is on stars massive enough ultimately to yield a neutron star, the same calculations are also valid for low mass systems. In the limiting scenarios of $m_{c}=0$ and $m_{c}=1$, we recover the well-studied cases of a $n=3 / 2$ polytrope and a point mass, respectively.

Figure 1 shows the pressure and density profiles as a function of radius for condensed polytropes with core masses $0,0.125$, and 0.5 . For comparison, we also display the profiles of red giant stars computed using the TWIN stellar evolution code (Eggleton 1971; Glebbeek, Pols, \& Hurley 2008) from the MUSE software environment $^{5}$ (Portegies Zwart et al. 2009): we evolve 10 and $25 M_{\odot}$ stars with initial helium abundance $Y=0.28$ and metallicity $Z=0.02$ until they obtain core masses of approximately $m_{c}=0.16$ and 0.27 , respectively. This corresponds to our initially $10 M_{\odot}$ star being at the very tip of the red giant branch and the initially $25 M_{\odot}$ star being on the upper part of the branch. The profiles of the simple condensed polytrope models follow the same general trend as those of the red giants, indicating that our simple models can indeed help provide an understanding of real red giant binaries.

Table 1 presents the models used in this paper. Column (1) gives the core mass $m_{c}$, while column (2) lists the corresponding $E_{\mathrm{O}}$ value: this is the parameter $E$ in the original notation of Osterbrock (1953), as well as in the notation of Härm \& Schwarzschild (1955) and Hjellming $\&$ Webbink (1987). (We prefer to reserve the variable $E$ for energy.) The value of $E_{\mathrm{O}}$ controls the shape of the density profile: to lowest order near the surface $(r \approx R)$, $\rho(r) \approx(2 / 5)^{3 / 2} E_{\mathrm{O}}(1-r / R)^{3 / 2} M /\left(4 \pi R^{3}\right)$. In practice, we adjust $E_{\mathrm{O}}$ to achieve the desired core mass $m_{c}$.

TABLE 1

Parent Star Characteristics

\begin{tabular}{cc}
\hline$m_{\mathrm{c}}$ & $E_{O}$ \\
$(1)$ & $(2)$ \\
\hline 0 & 45.4808 \\
0.05 & 39.9250 \\
0.1 & 35.2403 \\
0.125 & 33.1661 \\
0.15 & 31.2407 \\
0.175 & 29.4461 \\
0.2 & 27.7673 \\
0.25 & 24.7072 \\
0.3 & 21.9782 \\
0.4 & 17.2861 \\
0.5 & 13.3582 \\
0.6 & 9.9904 \\
0.7 & 7.0496 \\
0.8 & 4.4443 \\
0.9 & 2.1092 \\
0.99 & 0.1984 \\
\hline
\end{tabular}

We begin by making an SPH model of a single star

${ }^{5}$ http://muse.li 


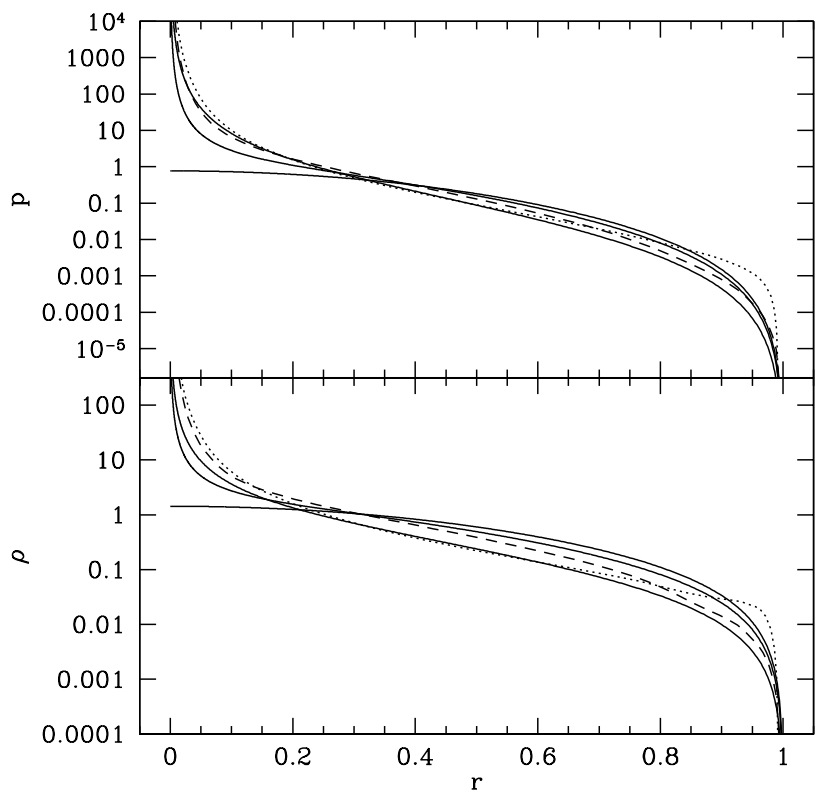

FIG. 1. - The solid curves show the quasi-analytic radial profiles of the pressure $p$ and density $\rho$ for $m_{c}=0,0.125$, and 0.5 condensed polytropes; for comparison, the dashed and dotted curves represent red giants, as modeled by the TWIN stellar evolution code, with initial masses respectively of 10 and $25 M_{\odot}$. For the condensed polytropes, curves associated with larger $m_{c}$ are higher on the left edge of the figure and lower on the right edge. Units are such that $G=M=R=1$.

in isolation. Unless stated otherwise, we use $N=$ 19938 SPH particles initially placed on a hexagonal close packed lattice with a lattice spacing constant $a_{1}=$ 0.0542 , with particles extending out to a radius that is between one and two smoothing lengths less than the full stellar radius. We model the stellar core as a point mass that interacts gravitationally, but not hydrodynamically, with the rest of the system, as suggested by Rasio \& Shapiro (1991) and others. The gravitational influence of these core points are softened according to the SPH kernel with $h=0.0498$. Particle masses are first apportioned according to the desired density profile and then slightly rescaled before relaxation begins to ensure that the correct total mass $M=1$ is precisely achieved. The entropic variable $A_{i}$ of each SPH particle is set to the desired polytropic constant $K$, which is determined from the mass-radius relation for $n=3 / 2$ condensed polytropes (Osterbrock 1953):

$$
R=(4 \pi)^{-2 / 3} G^{-1} K E_{\mathrm{O}}^{2 / 3} M^{-1 / 3} .
$$

After the initial parameters of the particles have been assigned, we relax the SPH fluid into hydrostatic equilibrium. This relaxation is effected by including an artificial viscosity contribution in the acceleration equation (with $\alpha=1$ and $\beta=2$ in equation (A19) of Gaburov et al. 2010), while still keeping $A_{i}$ constant for all particles. In this way, the entropy of the system is preserved while the system approaches equilibrium. The total energy typically decreases by less than a percent in the process, indicating that our initial assignment of particle properties was indeed very close to an equilibrium state.

This approach allows us to model the desired profiles
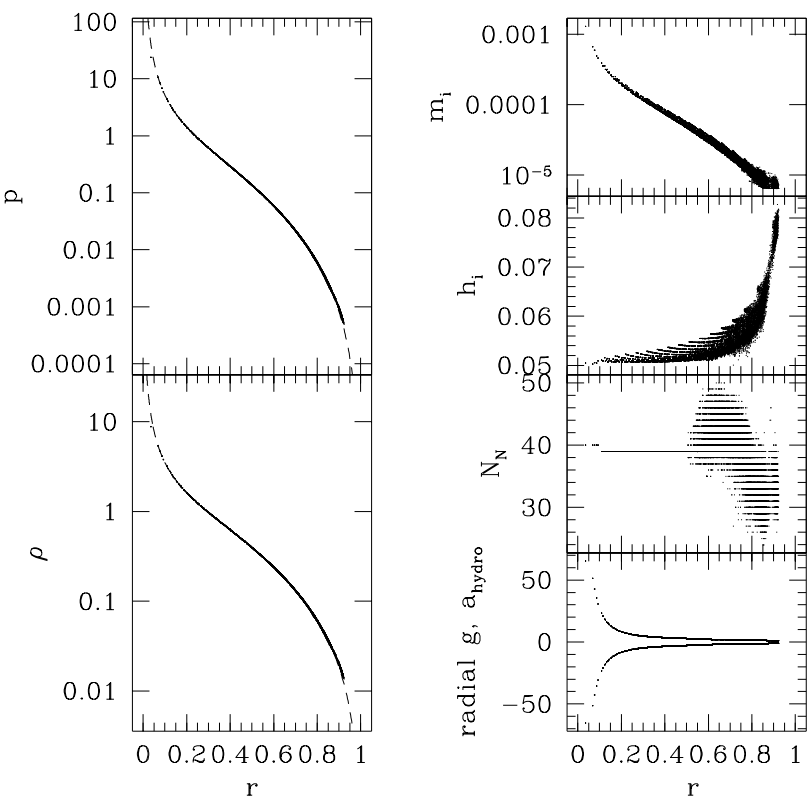

FIG. 2.- Properties of the model with core mass $m_{c}=0.25$ as a function of radius, after relaxation for 500 time units. The frames in the left column compare calculated pressure $p$ and density $\rho$ profiles of the star (dashed curves) against particle data from our SPH model (dots). The right column provides additional SPH particle data: individual SPH particle mass $m$, smoothing length $h$, number of neighbors $N_{N}$, and radial component of the hydrodynamic acceleration $a_{\text {hydro }}$ (upper data) and gravitational acceleration $g$ (lower data).

very accurately. An example is presented in Figure 2, where we plot desired profiles and SPH particle data for our relaxed $m_{c}=0.25$ star. Although the core and surface of the star of course cannot be resolved on the length scale of a smoothing length (typically 0.05 to 0.08 length units), the thermodynamic profiles of the SPH model nicely reproduce the quasi-analytic curves. Indeed, the SPH data in the left column of Figure 2 are difficult to distinguish from the desired pressure and density profiles throughout most of the star. We also note that the hydrodynamic and gravitational accelerations are very nearly equal in magnitude and opposite in direction, as necessary for hydrostatic equilibrium.

\subsection{Binary Equilibrium Configurations \\ 2.3.1. SPH Calculations}

The ability of our code to model close and contact binary systems for thousands of orbits or longer is presented in Gaburov et al. (2010). Here we present our methods for modeling equilibrium sequences of twin binaries, that is, binaries that consist of two identical stars in synchronized orbit. First, we place identical relaxed stellar models along the $x$ axis with their centers of mass separated by $r$. While the relaxation of the binary takes place, the entropic variable particle values are held constant. The center of mass of the entire system is fixed at the origin. In addition, the positions of the particles are continuously adjusted (by a simple uniform translation along the binary axis), so that the separation between the centers of mass equals the desired separation $r$.

The orbit is chosen to occur in the $x y$ plane. The an- 
gular velocity $\Omega_{\text {orb }}$ defining the corotating frame is updated at every timestep so that the centrifugal and inertial accelerations acting on the fluid cancel. In particular, we wish to find configurations in which $\Omega_{\text {orb }}^{2}\left(x_{i} \hat{\mathrm{x}}+\right.$ $\left.y_{i} \hat{\mathrm{y}}\right)=-\left(\dot{v}_{x, i} \hat{\mathrm{x}}+\dot{v}_{y, i} \hat{\mathrm{y}}\right)$, where the velocity derivatives on the right hand side are components of acceleration in the inertial frame. By taking the dot product with $m_{i}\left(x_{i} \hat{\mathrm{x}}+y_{i} \hat{\mathrm{y}}\right)$ and then summing over all particles, we obtain

$$
\Omega_{\mathrm{orb}}^{2}=\frac{-\sum_{i} m_{i}\left(x_{i} \dot{v}_{x, i}+y_{i} \dot{v}_{y, i}\right)}{\sum_{i} m_{i}\left(x_{i}^{2}+y_{i}^{2}\right)} .
$$

We also include a drag force that opposes the velocity and provides a contribution to the acceleration of $-\mathbf{v}_{i} / t_{\text {relax }}$. We use $t_{\text {relax }}=3$, approximately the fundamental period of oscillation for our parent models. We do not include any artificial viscosity contribution when finding binary equilibrium configurations.

In order to find equilibrium configurations for a precisely equal mass binary, even for configurations unstable to mass transfer, we enforce a symmetry in particle properties: for each particle $i$ in star 1 , there is a partner particle $j$ in star 2 at $x_{j}=-x_{i}$ and $y_{j}=-y_{i}$ with velocity components $v_{x, j}=-v_{x, i}, v_{y, j}=-v_{y, i}$ and with acceleration components $\dot{v}_{x, j}=-\dot{v}_{x, i}, \dot{v}_{y, j}=-\dot{v}_{y, i}$. All other properties are identical for any such pair of particles.

The separation $r$ between the centers of mass can be allowed to drift slowly so that an equilibrium sequence is constructed: a so-called "scanning run." In practice, we start runs that will scan over separations by holding the centers of mass fixed at an initial separation $r(0)$ for 40 time units, allowing the system to approach a tidally bulged equilibrium configuration. At an additional amount of time $t$, the separation is set according to $r(t)=r(0)\left[r\left(t_{\text {scan }}\right) / r(0)\right]^{t / t_{\text {scan }}}$. This form for $r(t)$ allows the change in $r$ to occur at a decreasing rate as the stars approach and interact more strongly, although the exact form is not critical to our results. We typically use $t_{\text {scan }}=300, r(0)=3.3$, and $r\left(t_{\text {scan }}\right)=2.1$.

\subsubsection{Data Reduction Methods}

Once an SPH binary calculation has completed, we analyze the system at various separations along the sequence. To this end, a useful quantity to consider is the effective potential, calculated as

$$
\Phi_{e}(x, y, z)=\Phi(x, y, z)-\frac{1}{2} \Omega_{\mathrm{orb}}^{2}\left(x^{2}+y^{2}\right),
$$

where $\Phi$ is the gravitational potential, the coordinate $y$ measures perpendicular to the binary axis in the orbital plane, and $z$ measures parallel to the rotation axis. Along the binary axis $(y=z=0)$, the effective potential has a local maximum $\Phi_{e}^{(i)}$ at $x=0$ (the inner Lagrangian point) and global maxima $\Phi_{e}^{(o)}$ at $|x|=x_{o}$ (the outer Lagrangian points). There are two minima at $|x|=x_{c}$, corresponding to the cores of the two components. ${ }^{6}$ In equilibrium, the fluid will fill up the effective potential well to some maximum, constant level $\Phi_{e}^{(s)}$. Borrowing the

\footnotetext{
${ }^{6}$ Note that in general $r \neq 2 x_{c}$, because we define the binary separation $r$ as the distance between the centers of mass of the two components.
}

terminology from models of W UMa binaries (Rucinski 1992, and references therein), we follow Rasio \& Shapiro (1995) and define the degree of contact $\eta$ as

$$
\eta \equiv \frac{\Phi_{e}^{(s)}-\Phi_{e}^{(i)}}{\Phi_{e}^{(o)}-\Phi_{e}^{(i)}} .
$$

Clearly, we have $\eta<0$ for detached configurations: that is, none of the fluid has a large enough effective potential energy to exceed the effective potential energy barrier at the inner Lagrangian point. For $0<\eta<1$, the effective potential of the fluid near $x=0$ does exceed the barrier, and the system is classified as a contact binary. For $\eta>1$, the envelopes overflow beyond the outer Lagrangian surface, and no dynamical equilibrium configuration can be achieved; that is, the system has exceeded the Roche limit. Calculations in which we slowly scan to smaller separations can therefore determine position of first contact $(\eta=0)$, the secular stability limit (at the minimum energy and angular momentum along the sequence), and the Roche limit $(\eta=1)$.

It is important to realize that the equilibrium sequence of a twin binary passes smoothly from detached to contact configurations as the separation $r$ decreases. This is in contrast to all binary equilibrium sequences with mass ratio $q \neq 1$, which terminate at a Roche limit corresponding to the onset of mass transfer through the inner Lagrangian point (that is, once one of the binary components overflows its Roche lobe). For twin binaries, however, the Roche limit, which we still define as the last equilibrium configuration along a sequence with decreasing $r$, corresponds to the onset of mass shedding through the outer Lagrangian points: as an example, note that several particles have been shed to the far left and far right of the $r=2.22$ frame in Fig. 3 .

We estimate $\Phi_{e}^{(s)}$ from our SPH models by finding the maximum effective potential of the points along the $x$ axis that are within one smoothing length of the center of an SPH particle. Thus, even if the centers of all SPH particles are within the outer Lagrangian surface, we may still consider the system as having reached the Roche limit when some smoothing kernels extend substantially beyond the outer Lagrangian surface. Such an estimate accounts for the fact that an SPH particle is not a point mass but instead represents a parcel of fluid with a density profile described by the smoothing kernel. Our means of estimating $\Phi_{e}^{(s)}$ allow our critical separation results to converge quickly to a steady value as the resolution is increased up to the resolution presented in this paper (see $\S 3.1$ ).

\subsection{Dynamical Calculations}

We generate initial conditions for our dynamical runs by taking a configuration at the desired separation $r$ from a scanning run and then relaxing for an additional 200 time units. If a particle escapes past an outer Lagrangian point during this time interval, then we end the relaxation stage and begin following the dynamics immediately. During dynamical calculations, we include no drag force and move the particles according to their velocities in the usual way (for details, see Gaburov et al. 2010). Particles are again treated independently so that mass transfer events can be followed; that is, unlike the scans 
described in $\S 2.3$, no symmetry constraints are applied to particle motion. Artificial viscosity is implemented in both the acceleration and the entropy equations. To minimize the spurious effects of artificial viscosity (Lombardi et al. 1999), our dynamical calculations are done in a rotating frame, with the the angular velocity $\Omega_{\text {orb }}$ calculated once at the beginning of the dynamical evolution and thereafter held constant when applying Coriolis and centrifugal forces.

\section{RESULTS}

Using the methods described above, we construct twin binary sequences for 16 different core masses $m_{c}$ listed in Table 1 and covering the range from 0 to 0.99 . In $\S 3.1$, we create an equilibrium sequence for each core mass by slowly scanning over the binary separation, thereby identifying the separations of first contact, of the secular instability (if it exists), and of the Roche limit. In $\S 3.2$, separate dynamical calculations of various initial separation $r$ then allow us to test the dynamical stability of the contact configurations and to follow any mass transfer and the merger in unstable systems.

\subsection{Equilibrium Sequences}

Representative snapshots along the $m_{c}=0.1$ equilibrium sequence are presented in Figure 3. The structure of these solutions is shown both in projection onto the orbital plane (the $x y$ plane) and in terms of the effective potential $\Phi_{e}$. The thick solid curves in Figure 3 are the surfaces of constant effective potential $\Phi_{e}$ that mark the inner and outer Lagrangian surfaces. For fixed $x, \Phi_{e}$ is minimum on the binary axis $(y=z=0)$, and this minimum value is given as a dashed curve in Figure 3. In hydrostatic equilibrium, the fluid fills up to a constant level $\Phi_{e}^{(s)}$ that is independent of $x$.

Referring to Figure 3 we see that at the initial separation in our scan, $r=3.30$, the system is tidally bulged and the binary is detached: the fluid does not extend out to the inner Lagrangian surface (also known as the Roche lobe). At a separation $r=2.71$, the binary stars fill the inner Lagrangian surface and make first contact through the L1 point, located at the origin. Once the separation decreases to $r=2.42$, the binary reaches the secular instability limit, as marked by a minimum in the energy $E$ and angular momentum $J$ along this equilibrium sequence (see below). The critical separation $r=2.37$ at which mass transfer commences is identified with dynamical calculations described in $\S 3.2$; in this and other scans through equilibrium configurations, however, we suppress the mass transfer by enforcing symmetry in particle properties (see §2.3.1). At a separation $r=2.24$, the fluid extends out to the outer Lagrangian surface, marking the Roche limit. At even smaller separations, for example at the separation $r=2.22$ shown in the figure, no equilibrium configurations exist and the stars shed mass through the outer Lagrangian surface near the L2 point. The variation of critical effective potentials and the degree of contact $\eta$ along this $m_{c}=0.1$ equilibrium sequence is illustrated in Figure 4. For future reference, we note the approximately linear dependence of $\eta$ on the separation $r$.

From Figure 5, we note that as $m_{c}$ increases toward 1 , our results approach the Keplerian solution of two orbiting point masses. As expected, deviations from the point
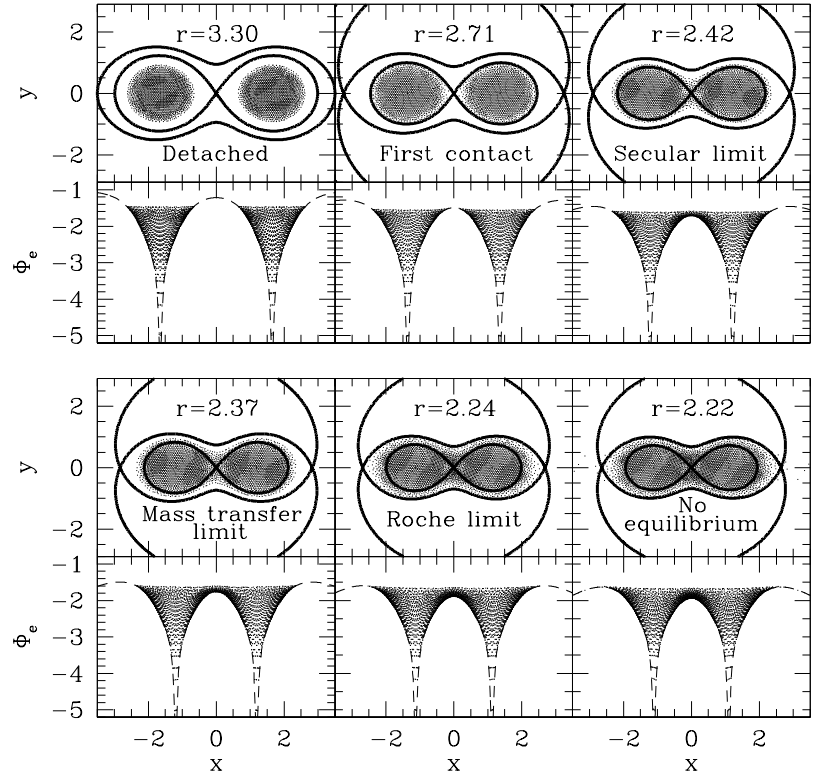

FIG. 3.- Sequence of binary equilibrium configurations for two identical condensed polytropes of core mass $m_{c}=0.1$. Projections onto the orbital plane (the $x y$ plane) are shown at six different binary separations for those SPH particles with $|z|<0.06$. The thick solid curves represent two surfaces of constant effective potential $\Phi_{e}$ (see eq. [4]): namely, the inner and outer Lagrangian surfaces passing through the points L1 and L2. Shown beneath each configuration are corresponding projections onto the $\left(x, \Phi_{e}\right)$ plane for the same particles. The dashed curves give the variation of $\Phi_{e}$ along the binary axis $(y=z=0)$. Contact configurations are obtained when the binary separation $r \lesssim 2.71$ (in units where an isolated binary component has radius $\widetilde{R}=1$ ). For $r \lesssim 2.24$, mass shedding through the outer Lagrangian points occurs, and no equilibrium configuration exists.

mass result increase as a given binary becomes more deeply in contact or as we consider a binary associated with a smaller core mass. From the bottom frame of Figure 5 , we note that smaller core masses (corresponding to stars with less centrally concentrated density profiles) have a smaller orbital period at any given separation. For $m_{c}<1$, tidal interactions between the two stars make the effective potential stronger than $1 / r$ and shorten the rotation period compared to a point mass system. For $m_{c}=0.1$, for example, the deviation of the orbital period from the point mass result is approximately $1 \%$ at first contact, $2 \%$ at the secular instability limit, and $3 \%$ at the Roche limit.

As in Rasio \& Shapiro (1995), we determine the secular stability limit along the equilibrium sequence by locating where both the total energy $E$ and total angular momentum $J$ are minimum in curves such as those of Figure 5. Our numerical results provide an accurate determination of this point for a close binary system, as the separate minima in $E$ and $J$ coincide to high numerical accuracy. This is in accord with the general property that $d E=\Omega_{\text {orb }} d J$ along any sequence of uniformly rotating fluid equilibria (Ostriker \& Gunn 1969).

For the $m_{c}=0$ binary, secular instability occurs soon after contact along this sequence and therefore stable, long-lived equilibrium configurations can exist only in shallow contact, $\eta \lesssim 0.2$. In contrast, the sequences with non-zero core masses permit much deeper contact before 


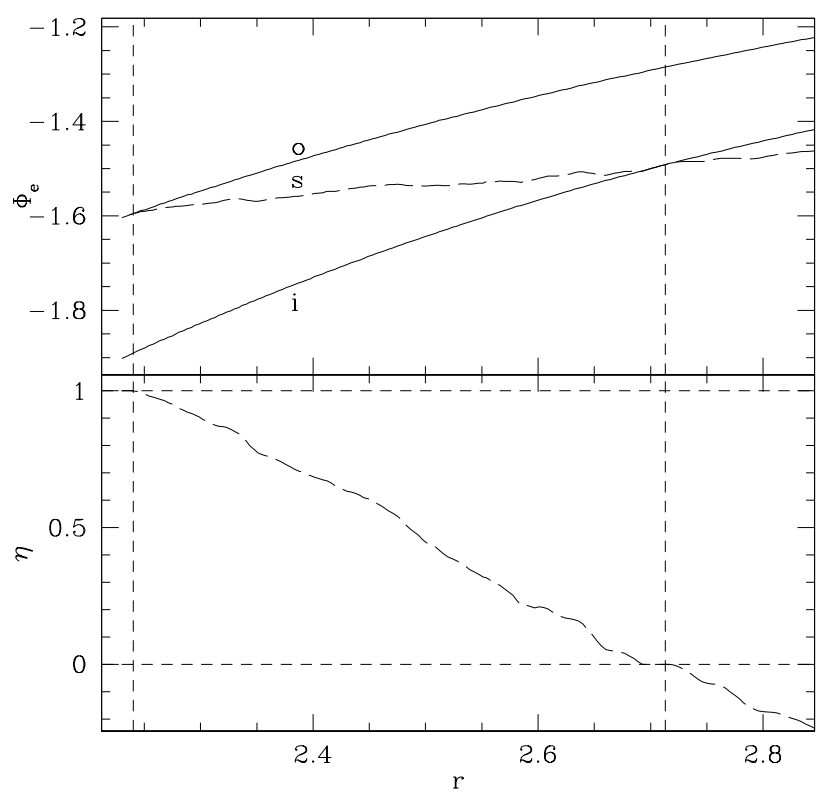

FIG. 4.- Variation of critical effective potentials along the equilibrium sequence presented in Fig. 3. Values of the effective potential at the outer Lagrangian surface (solid curve o), the inner Lagrangian surface (solid curve i), and the fluid surface (long dashed curve s) are shown as a function of binary separation $r$ in the top frame. The degree of contact $\eta$ (eq. [5]) is shown in the bottom frame as the long dashed curve. The short dashed curves give the positions of first contact $(\eta=0)$ and of the Roche limit $(\eta=1)$.

the secular instability is reached. For example, a binary with core masses of 0.125 does not reach the secular instability until nearly $\eta=0.9$. For core masses $m_{c} \gtrsim 0.15$, the stars will reach the Roche limit before the secular instability limit. Tables 2,3 , and 4 respectively present system properties at first contact $(\eta=0)$, the secular instability limit (where $E$ and $J$ are minima), and at the Roche limit $(\eta=1)$ for our sequences of various core mass $m_{c}$. Additional runs at varying resolution indicate that the results in our tables have converged to within $\sim 1 \%$ (e.g. see Fig. 6).

These results for critical separations are summarized in Figure 7. Due to tidal effects, the volume of each star is typically $1-2 \%$ larger at first contact than it is for that star in isolation. The separation $r_{\mathrm{fc}}$ at first contact is only weakly dependent on $m_{c}$, being within $2 \%$ of 2.7 for any core mass. For comparison, we note that the standard simple treatment of twin binaries would imply a first contact separation of $1 / 0.3799=2.63$, where the factor 0.3799 comes from numerical integration of Roche lobe volumes around identical point masses (e.g., Eggleton 1983) and any change in the volumes of the stars due to tidal effects is neglected. We see therefore that finite size effects act to increase the separation of first contact.

All three of the critical separations considered (first contact, secular instability, and Roche limit) tend to decrease as the core mass increases. It is straightforward to find fitted formulas for the critical separations that are accurate to within $\sim 1 \%$ for any core mass $m_{c}$ : for first contact

$$
r_{\mathrm{fc}} \approx 2.66+0.08\left(1-m_{c}\right)^{4}
$$

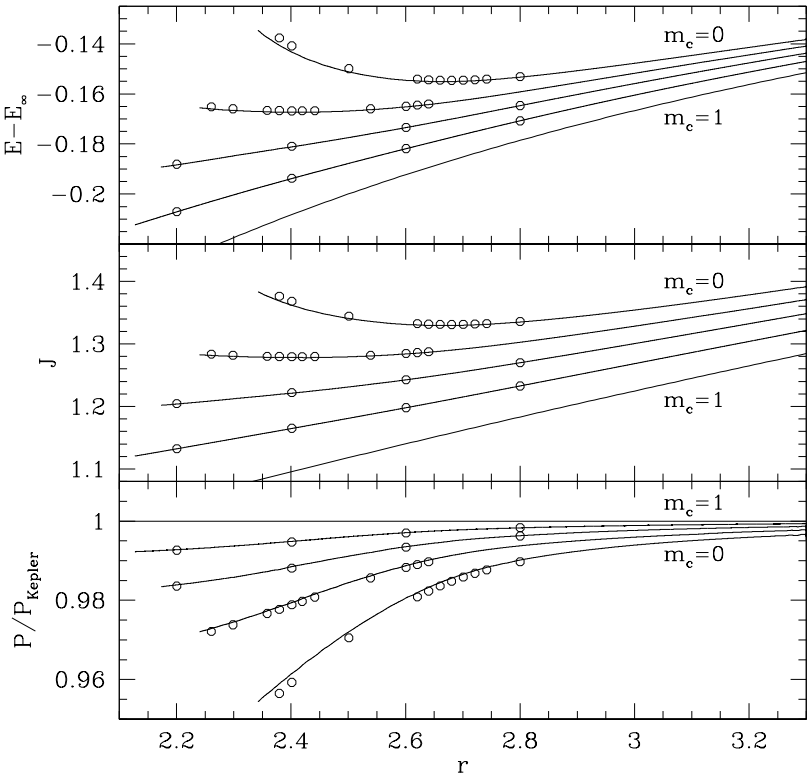

FIG. 5.- Variation of the system energy $E$ (relative to the total self-energy $E_{\infty}$ of the binary components at infinity), angular momentum $J$, and orbital period $P$ along the equilibrium sequence for twin binaries with $m_{c}=0,0.1,0.25,0.5$, and 1 . In the $E$ and $J$ frames, higher curves correspond to smaller core mass, while in the $P$ frame higher curves correspond to larger core mass. The orbital period $P$ is normalized to the analytic point mass result $P_{\text {Kepler }}=2^{1 / 2} \pi r^{3 / 2}$. The curves are from SPH scans of the equilibrium sequence, except for the $m_{c}=1$ curve which is the analytic result for two point masses. The $m_{c}=0$ and 0.1 curves exhibit a minimum in $E$ and $J$, marking the position of the secular instability limit. The curves from the SPH scans terminate at the Roche limit, where mass shedding through the outer Lagrangian point commences. Critical points along our SPH scans are presented in Tables 2, 3, and 4. The individual data points (open circles) in this figure result from relaxing for an additional 200 time units at the given separation $r$. The agreement between these points and their corresponding scan helps to confirm that our scans are indeed producing equilibrium sequences.

TABLE 2

FIRST CONTACT ALONG THE EQUILIBRIUM SEQUENCES OF TWIN BINARIES ${ }^{a}$

\begin{tabular}{ccccc}
\hline$m_{c}$ & $r$ & $P$ & $E-E_{\infty}$ & $J$ \\
\hline 0.000 & 2.75 & 20.1 & -0.154 & 1.33 \\
0.050 & 2.73 & 19.8 & -0.159 & 1.31 \\
0.100 & 2.72 & 19.7 & -0.162 & 1.29 \\
0.125 & 2.72 & 19.8 & -0.163 & 1.29 \\
0.150 & 2.71 & 19.7 & -0.165 & 1.28 \\
0.175 & 2.69 & 19.5 & -0.167 & 1.27 \\
0.200 & 2.69 & 19.5 & -0.168 & 1.27 \\
0.250 & 2.69 & 19.5 & -0.170 & 1.25 \\
0.300 & 2.70 & 19.6 & -0.171 & 1.25 \\
0.400 & 2.68 & 19.4 & -0.175 & 1.23 \\
0.500 & 2.66 & 19.3 & -0.178 & 1.21 \\
0.600 & 2.67 & 19.3 & -0.180 & 1.20 \\
0.700 & 2.66 & 19.2 & -0.183 & 1.18 \\
0.800 & 2.67 & 19.4 & -0.184 & 1.17 \\
0.900 & 2.67 & 19.4 & -0.186 & 1.16 \\
0.990 & 2.70 & 19.7 & -0.185 & 1.16 \\
\hline
\end{tabular}

${ }^{\mathrm{a}}$ Units are defined such that $G=M=R=1, m_{c}$ is the core mass of each component, $r$ is the binary separation, $\eta$ is the degree of contact (eq. [5]), $P$ is the orbital period, and $E-E \infty$ and $J$ are the orbital energy and angular momentum, respectively; the energy $E_{\infty}$ is the total equilibrium energy at infinite separation (that is, twice the energy of a single component in isolation). 
TABLE 3

SECULAR INSTABILITY ALONG THE EQUILIBRIUM SEQUENCES OF TWIN BINARIES ${ }^{\mathrm{b}}$

\begin{tabular}{cccccc}
\hline$m_{c}$ & $r$ & $\eta$ & $P$ & $E-E_{\infty}$ & $J$ \\
\hline 0.000 & 2.67 & 0.17 & 19.1 & -0.155 & 1.33 \\
0.050 & 2.55 & 0.40 & 17.7 & -0.161 & 1.30 \\
0.100 & 2.42 & 0.64 & 16.4 & -0.167 & 1.28 \\
0.125 & 2.29 & 0.86 & 15.1 & -0.171 & 1.26 \\
0.150 & 2.22 & 1.0 & 14.3 & -0.175 & 1.25 \\
\hline
\end{tabular}

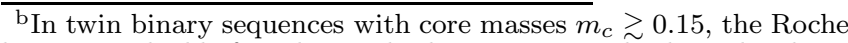
limit is reached before the secular limit. Units and column headings are as in Table 2 , footnote a; the degree of contact $\eta$ is defined by eq. 5 .

TABLE 4

ROCHE LIMIT ${ }^{c}$ ALONG THE EQUILIBRIUM SEQUENCES OF TWIN BINARIES ${ }^{d}$

\begin{tabular}{ccccc}
\hline$m_{c}$ & $r$ & $P$ & $E-E_{\infty}$ & $J$ \\
\hline 0.000 & 2.34 & 15.2 & -0.135 & 1.38 \\
0.050 & 2.28 & 14.7 & -0.153 & 1.32 \\
0.100 & 2.24 & 14.5 & -0.166 & 1.28 \\
0.125 & 2.22 & 14.3 & -0.171 & 1.27 \\
0.150 & 2.21 & 14.3 & -0.175 & 1.25 \\
0.175 & 2.20 & 14.2 & -0.179 & 1.24 \\
0.200 & 2.19 & 14.1 & -0.183 & 1.22 \\
0.250 & 2.17 & 14.0 & -0.189 & 1.20 \\
0.300 & 2.16 & 13.9 & -0.195 & 1.18 \\
0.400 & 2.14 & 13.8 & -0.204 & 1.15 \\
0.500 & 2.13 & 13.7 & -0.212 & 1.12 \\
0.600 & 2.12 & 13.6 & -0.219 & 1.10 \\
0.700 & 2.12 & 13.6 & -0.224 & 1.08 \\
0.800 & 2.10 & 13.5 & -0.231 & 1.05 \\
0.900 & 2.10 & 13.5 & -0.235 & 1.04 \\
0.990 & 2.12 & 13.7 & -0.236 & 1.03 \\
\hline
\end{tabular}

${ }^{\mathrm{c}}$ Defined as the equilibrium configuration with the minimum binary separation.

${ }^{\mathrm{d}}$ Unit and column definitions are identical to those in Table 2, footnote a.

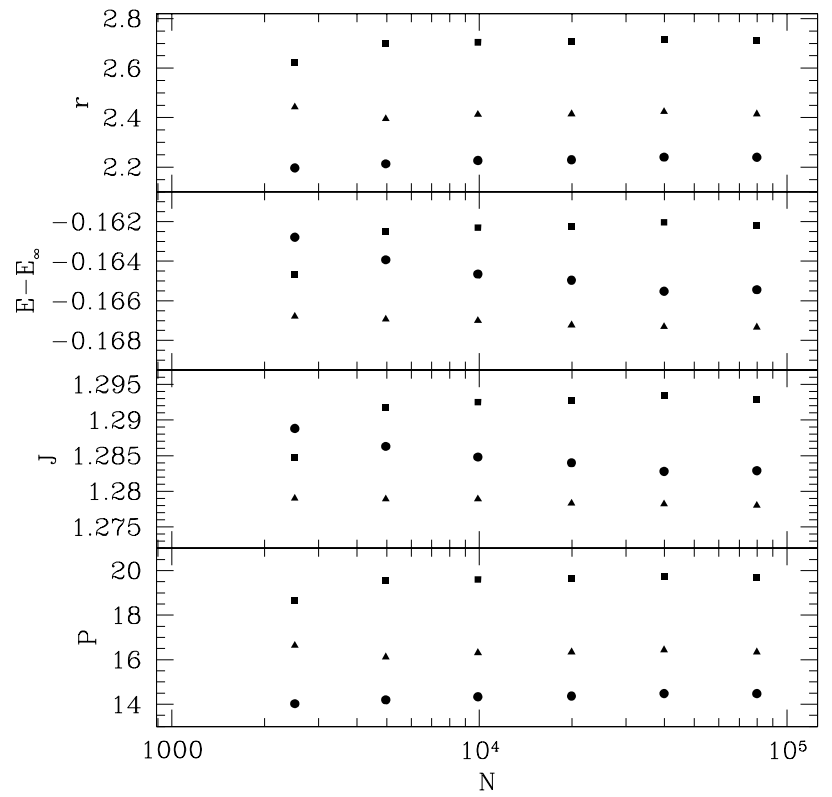

FIG. 6.- Critical separation $r$, energy $E-E_{\infty}$, angular momentum $J$, and orbital period $P$ at first contact (squares), secular instability (triangles), and the Roche limit (circles) versus total particle number $N$ for several $m_{c}=0.1$ equilibrium scans. Note the convergence of results for $N \gtrsim 10^{4}$. Most of the binary calculations in this paper employ $N \approx 4 \times 10^{4}$ particles.

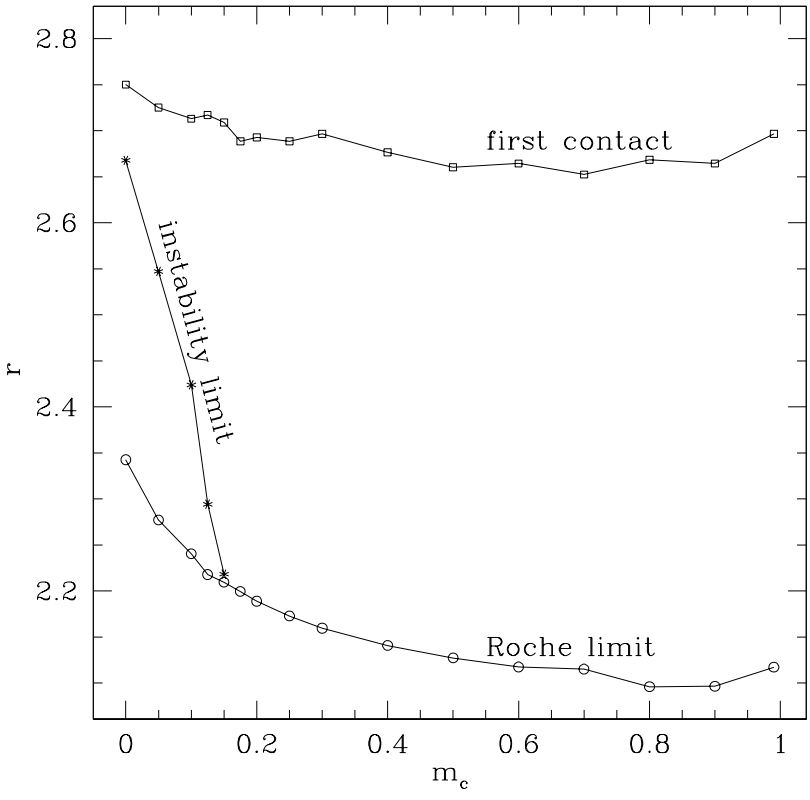

FIG. 7.- Separation at first contact (squares), the onset of secular instability (stars), and the Roche limit (circles) versus core mass $m_{c}$. The data points are determined from SPH scans of equilibrium sequences, as reported in Tables 2,3 , and 4 . The lines are simply to help guide the eye.

and for the secular instability limit

$$
r_{\mathrm{sec}} \approx 2.69-3 m_{c} \text {. }
$$

We note that the $1-m_{c}$ in equation (6) equals the envelope mass (in units where the total stellar mass $M=1$ ). We give our fit for the Roche limit separations in the next subsection, where we can determine these data with slightly better accuracy. We have not fit for the slight increase in the first contact data as the core mass is increased from $m_{c}=0.9$ to 0.99 , as this feature is a numerical artifact due to our $m_{c}=0.99$ single star equilibrium model settling to a radius a few percent larger than 1 .

The critical core mass $m_{c} \approx 0.15$, for which the secular instability and Roche limits coincide, can be determined graphically from Figure 7 by extrapolating the line connecting the secular instability data down to the Roche limit curve. This intersection is important because it implies that all of our equal mass binaries with $m_{c} \gtrsim 0.15$ can stably exist in deep contact, at separations all the way down to the Roche limit. Thus, essentially all twin red giant binaries will coalesce only due to mass shedding through the outer Lagrangian points. In contrast, twin binaries with $m_{c} \lesssim 0.15$, corresponding primarily to main-sequence stars and subgiants, reach the secular instability limit at a larger separation than that of the Roche limit. As we will see in the next subsection, the secular instability limit is usually accompanied by $d y$ namical mass transfer at the same or a slightly smaller separation. Therefore, main sequence and subgiant twin binaries, as contrasted to most red giant twins, will start coalescing (a) when in more shallow contact and (b) through mass transfer across the inner Lagrangian point.

\subsection{Dynamical Integrations}




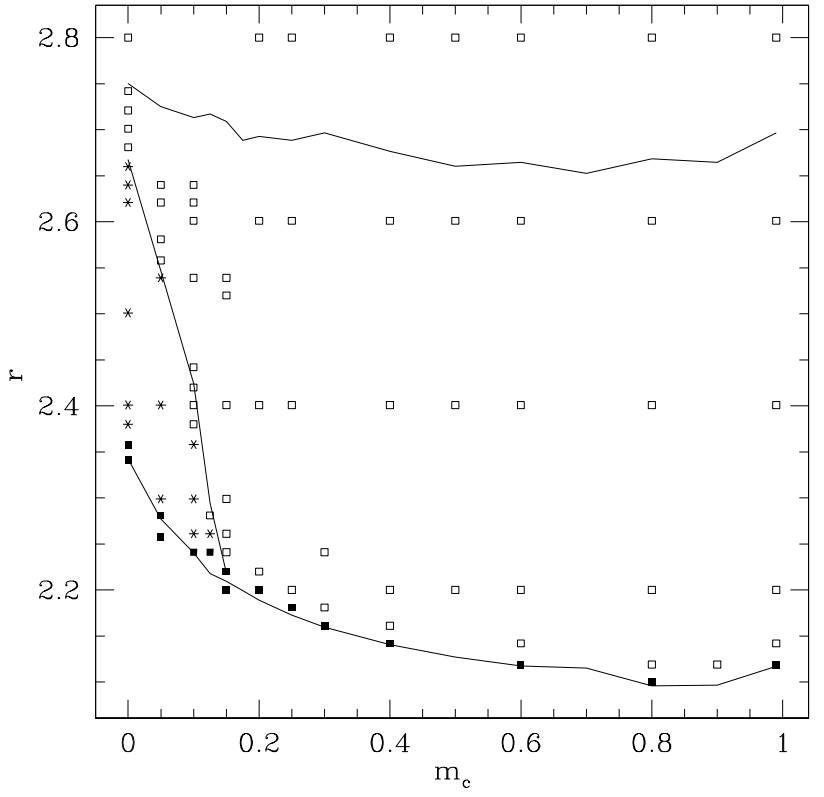

FIG. 8.- Results of dynamical integrations: stable binaries (open squares), dynamically unstable binaries (asterisks), and configurations with no equilibrium (filled squares). The curves represent the critical separations as determined by scanning runs, as in Fig. 7. The agreement between the results of the equilibrium sequences and dynamical calculations is excellent.

We now study the stability of binary configurations with fully dynamical SPH integrations (see $\S 2.4$ for details of the setup). Figure 8 summarizes the results of nearly 100 dynamical simulations with various $m_{c}$ and initial $r$ values. $^{7}$ We find the Roche limit to be very nearly at the separations determined from the equilibrium scans, and the additional relaxation that we perform before beginning a dynamical calculation allows us to determine these separations even more accurately. In addition, we find that most systems that are secularly unstable are also dynamically unstable to mass transfer and then merger, as discussed below.

The time evolution of the separation of $m_{c}=0.05$ twin binaries is illustrated in Figure 9 for several different initial separations. This figure also indicates the secular instability limit $r_{\text {sec }}=2.547$, as determined by the energy and angular momentum minima in the equilibrium sequence of these binaries (see §3.1). Systems with separations $r \gtrsim r_{\mathrm{sec}}$ are clearly dynamically stable, while those with $r \lesssim r_{\text {sec }}$ are dynamically unstable. That is, the secular and dynamical stability limits coincide or very nearly coincide, at least at this core mass.

The bottom plot of Figure 9 shows the dynamical evolution of two cases that straddle the instability limit. For the $r=2.56$ case, an epicyclic period of 350 time units is clearly evident, much larger than the orbital period of 17.9 time units. The large difference in these periods is an indication of how close the system is to an instability limit (Rasio \& Shapiro 1994). Indeed, if $r$ were precisely at the dynamical stability limit, the period of small epicyclic oscillations would formally be infinite.

7 Visualizations of selected simulations are available at http://webpub.allegheny.edu/employee/j/jalombar/movies/.
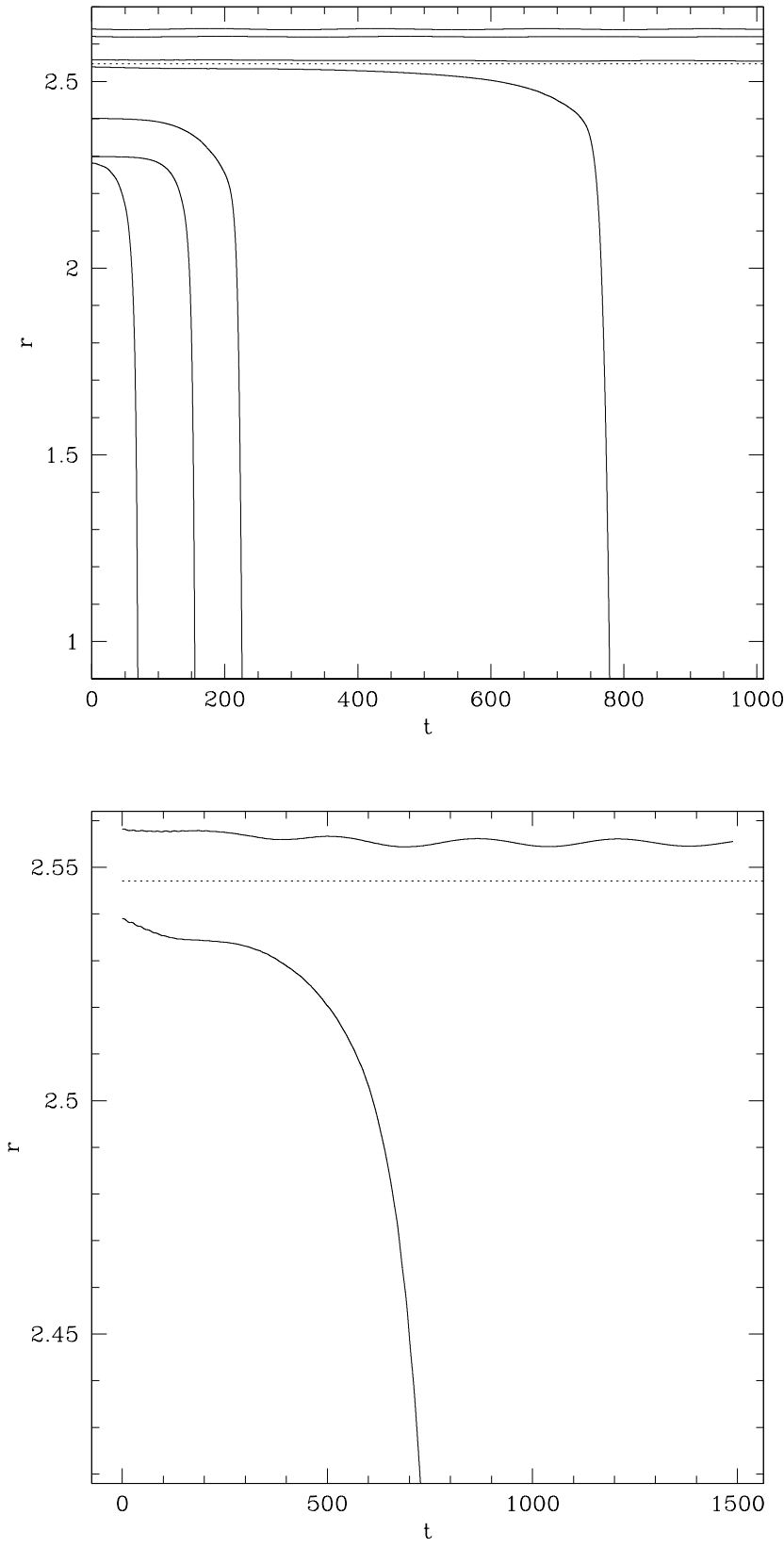

Fig. 9.- Top plot: Separation $r$ versus time in several different dynamical SPH calculations for the core mass $m_{c}=0.05$ twin binaries. The initial separations are $r=2.64,2.62,2.56,2.54$, 2.40, 2.30 and 2.28. Bottom plot: Zoomed in view of the $r=2.56$ and 2.54 cases, which straddle the instability limit. In both plots, the horizontal dotted line represents the secular instability limit at $r=2.547$, as identified by an equilibrium scan.

Figure 10 presents projected particle positions (top) and column density plots (bottom) at six different times in the $m_{c}=0.05, r=2.54$ dynamical calculation, a case just inside the instability limit. Colors in the particle plot are used to indicate from which component the particles originated. The coordinate system used here rotates counterclockwise with a period of 17.64 time units, which equals the orbital period of this binary at early times, before significant mass transfer has occurred. The instability initially manifests itself in the form of a nar- 
row arm of gas that begins in the outer layers of one star, gradually flows across the neck surrounding the inner Lagrangian point, and then creeps around the other star (see the $t=746$ and 771 particle plot frames). The mass transfer drives the binary components closer, triggering the excretion of mass through the outer Lagrangian points $(t=771)$ and accelerating the inspiral of the two cores. By $t=784$, the mass transfered from one star has completely engulfed the other. At later times, the merger product approaches a rapidly rotating, axisymmetric configuration centered on the two cores orbiting in a tight binary (see the $t=792$ and 961 frames).

Figure 11 shows the evolution of binary separation for $m_{c}=0.2$ cases. Recall from $\S 3.1$ that there is no secular instability limit at this core mass. Instead, stable equilibrium models exist all the way to the Roche limit. A binary at an initial separation $r=2.22$ (or larger) orbits stably. In contrast, a binary at $r=2.20$ gradually loses mass through the outer Lagrangian points, triggering a stage of rapid coalescence. The period of mass loss persists for several orbital periods: each of the oscillations superposed on the decreasing $r=2.20$ curve in the bottom plot of Fig.11 corresponds to one orbital period. These dynamical calculations indicate that the Roche limit for a $m_{c}=0.2$ twin binary indeed occurs near $r=2.2$, in excellent agreement with the $r=2.19$ critical value estimated from the equilibrium scan.

Figure 12 presents both particle positions and column density plots at six different times in the $m_{c}=0.2$, $r=2.20$ dynamical calculation, a case just inside the Roche limit. The coordinate system used here rotates counterclockwise with a period of 14.22 time units, which equals the orbital period of this binary at early times. Gas is excreted almost immediately, with each parcel of gas carrying a specific angular momentum essentially equal to that of the outer Lagrangian points. In contrast to mergers with $m_{c} \lesssim 0.15$, the excreted gas originates equally from both binary components and flows past the outer Lagrangian points symmetrically. As the outer Lagrangian points are the outermost positions at which gas can be in static equilibrium, they are also the positions of largest possible specific angular momentum in rigidly rotating equilibrium twin binaries. Consequently, the mass loss necessarily decreases the average angular momentum per unit mass of the gas remaining within the outer Lagrangian surface, causing the binary components, along with their cores, to inspiral: see the appendix of Webbink (1976) for a rigorous analysis of the angular momentum budget during mass excretion. As the components get closer, the excretion rate increases, and in addition the resulting arms become more tightly wound (compare the $t=202$ frame to later ones). By $t=233$, the central regions of the binary components have effectively merged. At later times, the merger product approaches a rapidly rotating, axisymmetric configuration (see Fig. 13).

Figure 14 shows energies versus time for the $m_{c}=0.2$, $r=2.20$ calculation. The kinetic energy $T$ gradually increases as the binary components inspiral, until the cores approach closely at $t \approx 230$. The ensuing shocks cause the gas to expand, causing an overall decrease in the internal energy $U$ and increase in the gravitational potential energy $W$. The rapid variations in $T$ and $W$ at late times are due to the eccentric orbit of the central double core. The total energy $E$ is well conserved in this simulation, varying by only $0.4 \%$ from its minimum to maximum values over the entire time interval shown in Figure 14. Energy conservation in other runs is typically at least this good and often even much better. In our simulations, most of the small non-conservation in energy occurs at late times once the core particles have entered a tight orbit.

In all our merger simulations of twin binaries with fractional core masses of 0.15 or larger, each star loses mass through an outer Lagrangian point. Most of this mass ultimately ends up in a circumbinary envelope gravitationally bound to the central binary of cores. The mass ejected varies from $\sim 0.5 \%$ (for $m_{c}=0.15$ ) up to $\sim 7 \%$ (for $m_{c}=0.8$ ) of the total system mass. A trend evident from the simulations is that more massive cores ultimately remain more widely separated, after inspiraling, than less massive cores. The top frame of Figure 15 provides a closer look at how the separation of the cores evolves in several dynamical simulations that begin just inside the Roche limit. For very large fractional core masses $\left(m_{c} \gtrsim 0.9\right)$, the gas is simply not massive enough to affect significantly the dynamics of the cores: although they inspiral, they stay separated at distances on the order of the initial stellar radius. For moderately large core masses $\left(0.5 \lesssim m_{c} \lesssim 0.9\right)$, the cores inspiral to a fraction of a stellar radius, although the process halts at separations large enough still to be resolved by our simulations. For core masses in the range $0.15 \lesssim m_{c} \lesssim 0.5$, which corresponds to most red giants in nature, the cores rapidly inspiral to separations less than 0.1. Although our code has no mechanism for merging the cores, we do not expect such an effect to be relevant here: the size of a core relative to the stellar radius is typically only $\sim 10^{-4}$ or less for a giant, so that a tremendous amount of angular momentum would have to be removed from the double core before they could merge.

The bottom frame of Figure 15 concerns fractional core masses less than 0.15 , namely those cases that reach the secular instability limit before the Roche limit. The cores in these merger simulations inspiral to a separation $\lesssim 0.01$, considerably less than the spatial resolution. Whether or not the cores would merge in such circumstances will likely depend on the details of the parent star structure, with simulations that resolve the cores necessary to address the issue fully. We find that less than $0.5 \%$ of the system mass is ejected whenever the merger is triggered by mass transfer.

Figure 16 helps to quantify the entropy evolution during mergers by plotting the mass-average $<\ln A>$ over time for several cases. We note that, for our polytropic equation of state and gas of uniform composition, the specific entropy of a parcel of gas is proportional to $\ln A$ plus a constant. From the curves of Figure 16, we see that the change in entropy per unit mass tends to be larger, and develops on a longer timescale, for cases involving larger core masses. We also note that the entropy is still gradually increasing even at the end of our simulations, due to the influence of the central binary embedded within the system.

Like the critical separations for first contact and secular instability, the Roche limit separation tends to decrease as the core mass increases. A fitted formula consistent with our dynamical integrations to within $\sim 1 \%$ 

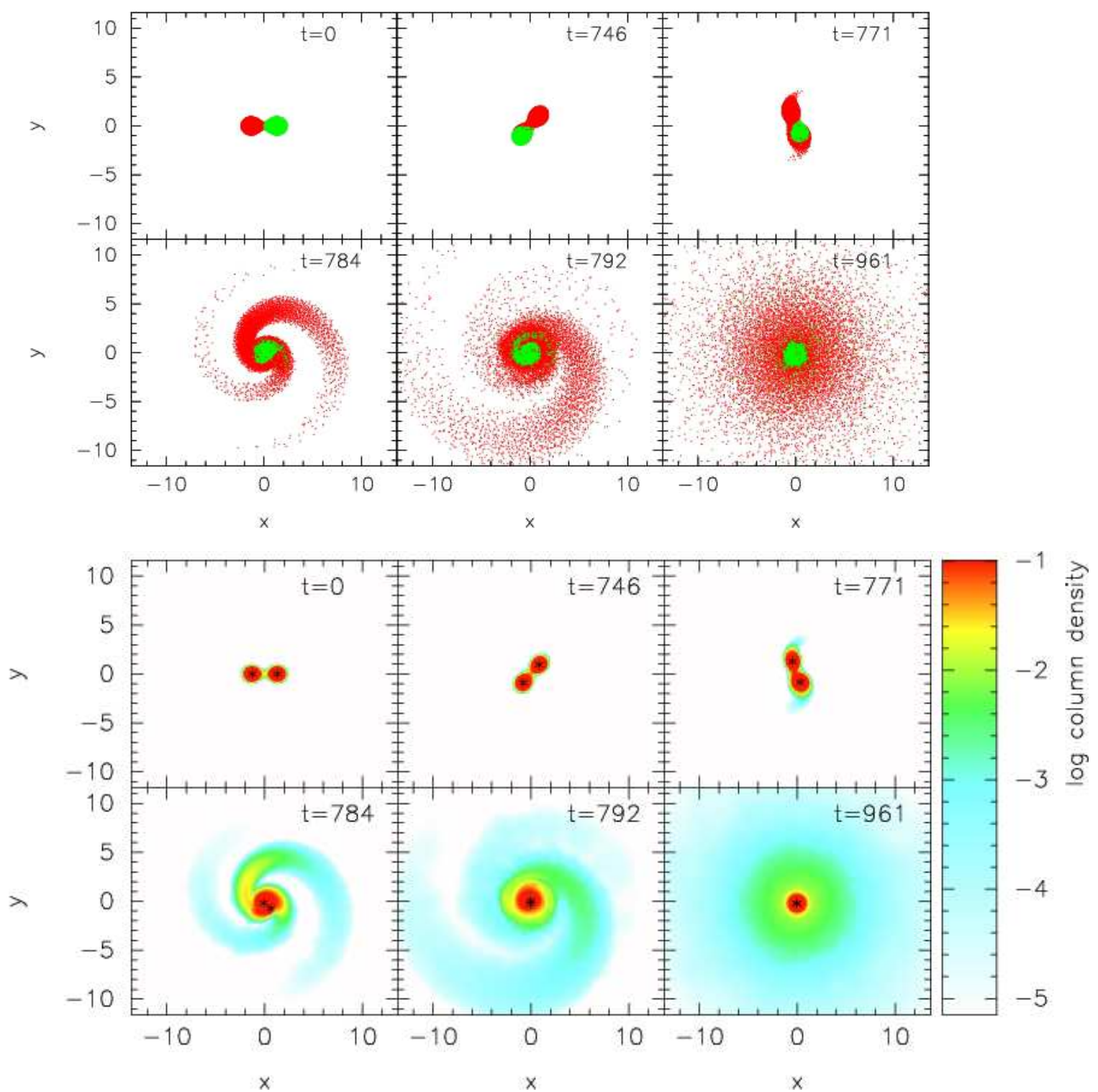

FIG. 10.- Top frames: Projection of SPH particles onto the orbital plane at six different times in the merger of a $m_{c}=0.05$ binary with initial separation $r=2.54$. Particles are colored according to the star in which they originated. Bottom frames: Column density plots at the same six moments, with the asterisks representing the positions of the compact cores.

for any core mass $m_{c}$ is

$$
r_{\text {Roche }} \approx 2.11+0.25\left(1-m_{c}\right)^{4} .
$$

Because the degree of contact $\eta$ varies nearly linearly with separation $r$ (for two examples, see Fig. 5 of this paper and Fig. 2 of Rasio \& Shapiro (1995)), this formula, along with others from $\S 3.1$, allows us also to estimate the degree of contact $\eta$ at the secular instability limit: $\eta_{\mathrm{sec}} \approx\left(r_{\mathrm{fc}}-r_{\mathrm{sec}}\right) /\left(r_{\mathrm{fc}}-r_{\text {Roche }}\right)$.

\section{DISCUSSION AND FUTURE WORK \\ 4.1. Summary of Main Results}

We have determined equilibrium sequences and performed dynamical calculations of twin binaries, focusing primarily on configurations in which the stars are in contact. Our equilibrium sequences of $\S 3.1$ allow us to determine the binary separation at first contact and at the innermost stable circular orbit as a function of the frac- tional core mass $m_{c}$. For $m_{c} \lesssim 0.15$, the innermost stable orbit occurs at the secular instability limit (marked by a minimum in energy and angular momentum along the equilibrium sequence); for $m_{c} \gtrsim 0.15$, the innermost stable orbit occurs at the Roche limit (defined as the minimum separation for which an equilibrium configuration exists). Our dynamical calculations of $\S 3.2$ confirm these critical separations and also reveal how the components inspiral once a binary passes the innermost stable orbit.

Figure 17 summarizes graphically our most basic results. Recall that the separation $r$ on the vertical axis is scaled to the unperturbed stellar radius $R$, while the core mass $m_{c}$ on the horizontal mass is scaled to the stellar mass $M$. Thus, as the components in a twin binary expand and gradually increase their core masses due to stellar evolution, the corresponding position in the parameter space of Figure 17 will shift down and slightly to the right. When this position drops below the top curve, which marks first contact, the binary enters the 

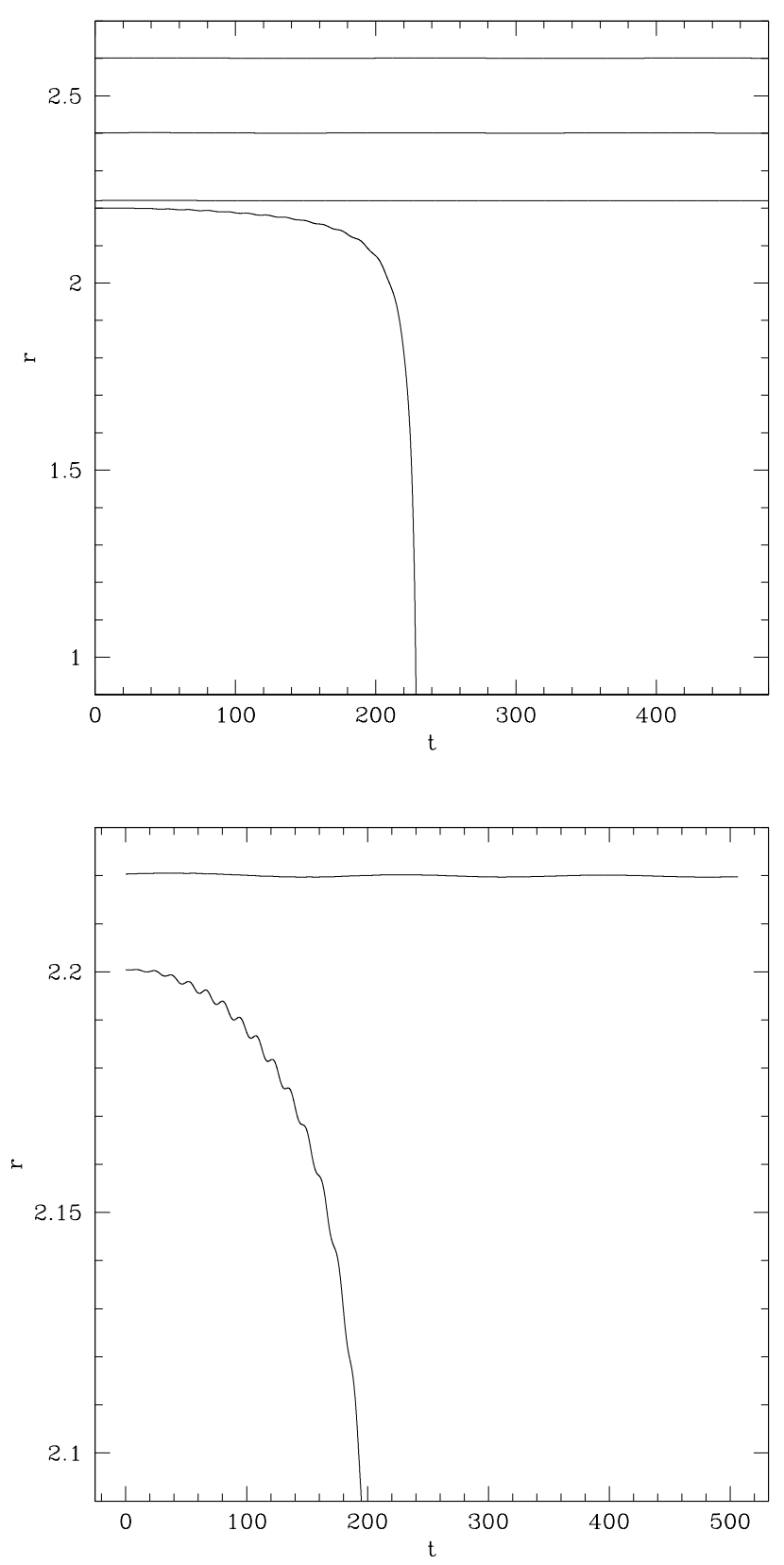

FIG. 11. - Like Fig. 9, but for twin binaries with core mass $m_{c}=0.2$. At this core mass, there is no secular instability limit: instead, the system become unstable only once it reaches the Roche limit at $r \approx 2.2$. The initial separations shown in the top plot are $r=2.60,2.40,2.22,2.20$, while a zoomed in view of the $r=2.22$ and 2.20 curves are shown in the bottom plot.

stable contact phase. When the position drops into either the unstable or no equilibrium portions of parameter space, the components merge.

The curves shown in Figure 17 are given by equations (6), (7), and (8); population modelers can use these fitted formulae in treatments of twin binaries. Consider, for example, such a binary with a given orbital separation $a$. The stellar evolution of each component gives the time dependence of the stellar radius $R$, the stellar mass $M$, and the core mass $M_{c}$. The dimensionless separation $r=a / R$ and the fractional core mass $m_{c}=M_{c} / M$ are thus known functions of time, and the evolutionary track can be placed in the theoretical $r$ versus $m_{c}$ diagram (Figure 17) to determine the final fate of the system.

We note that the volume of parameter space where real binaries would ultimately end up in the stable contact region, without crossing the instability limit or the Roche limit, is small. Such a situation would require a fine tuning of the initial semimajor axis $a$. For example, a star with an initial mass of $8 M_{\odot}$ will expand to $R \approx$ $370 R_{\odot}$ and reach a fractional core mass of $m_{c} \approx 0.2$ as it ascends the asymptotic giant branch, according to calculations by the TWIN stellar evolution code. For this core mass, $r_{\mathrm{fc}} \approx 2.7$ and $r_{\text {Roche }} \approx 2.2$. Thus, a twin binary composed of such stars will remain detached if $a \gtrsim$ $r_{\mathrm{fc}} R \approx 1000 R_{\odot}$ and will ultimately surpass the Roche limit if $a \lesssim r_{\text {Roche }} R \approx 800 R_{\odot}$. Only if $800 R_{\odot} \lesssim a \lesssim$ $1000 R_{\odot}$ will the binary reach the contact phase without the cores also inspiraling to form a tight binary.

The dynamic simulations of $\$ 3.2$ always start from a symmetric equilibrium configuration, with the binary components being hydrostatic in the corotating frame. In our simulations with core masses of $m_{c}=0.125$ and less, we find a dynamical instability to exist at or slightly inside the secular instability limit. This dynamical instability is a global instability of the equilibrium state, triggered by small numerical noise and characterized by a growing asymmetric mode. Binaries that come inside this instability limit first transfer mass gradually from one component to the other and eventually coalesce quickly as mass is lost through the outer Lagrangian points. The cores are left in a tight binary surrounded by a circumbinary envelope.

\subsection{Comparison with Other Works}

The merger of our small core mass twin binaries proceeds in a fashion qualitatively similar to that of the Q1.3 model of D'Souza et al. (2006). In that model, the binary is composed of purely polytropic components $\left(m_{c}=0\right)$ with mass ratio (donor to accretor) $q=1.3$. In both our simulations and theirs, the dynamical instability manifests itself as a gradually developing mass transfer flow, followed by excretion of gas through the outer Lagrangian points and merger of the stellar envelopes. One important difference, however, is that the instability in our $m_{c}=0$ case does not develop until the stars have reached a degree of contact $\eta \approx 0.17$, whereas the instability is present in the Q1.3 model while the binary is still semidetached. This difference highlights the stabilizing influence of the common envelope in twin binaries, even though the instability still exists even for $q=1$.

We can directly compare our results for the limiting case $m_{c}=0$ with those of Rasio \& Shapiro (1995). The agreement in the first contact, secular instability, and Roche limit separations is excellent (better than $1 \%$ ). The computational resources of the time, however, limited Rasio \& Shapiro (1995) to follow up to only $\sim 3$ orbits, so that they were unable to identify weak mass transfer events, that is, events that develop gradually over many dynamical timescales (Paczyiński \& Sienkiewicz 1972). As a result, they determined the dynamical instability limit to be at $r \approx 2.45$, well inside the secular instability limit. In contrast, by following the dynamical evolution of $m_{c}=0$ twin binaries for up to $\sim 100$ orbits, we find that the dynamical instability limit 

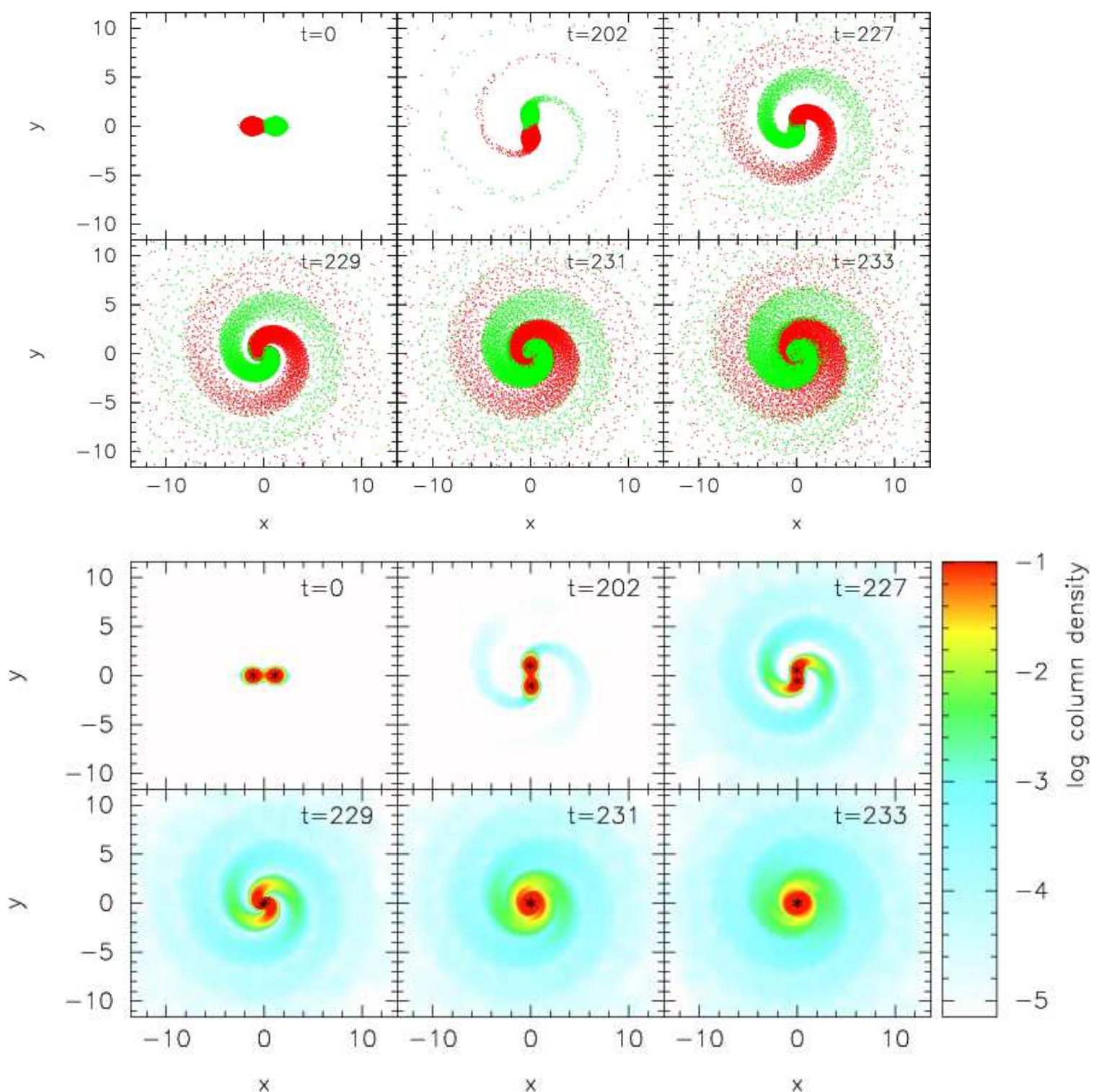

Fig. 12. - Like Fig. 10, but for the $m_{c}=0.2$ dynamical calculation starting at $r=2.20$, just inside the Roche limit.

actually coincides, or nearly coincides, with the secular instability limit at $r \approx 2.67$.

\subsection{Relevance to Binary Neutron Stars}

We find that twin binaries with $m_{c} \gtrsim 0.15$ exist stably at separations all the way down to the Roche limit, where mass is then excreted symmetrically through the outer Lagrangian points. This excretion carries away angular momentum and causes the stars, along with their cores, to inspiral on a dynamical timescale. For core masses $m_{c} \lesssim 0.9$, the cores inspiral to a final separation that is a fraction of the stellar radius. Indeed for $m_{c} \lesssim 0.5$, which corresponds to most giant stars in nature, the final separation of the cores is less than one-tenth the stellar radius. Thus, we are left with two cores in a tight binary surrounded by the combined gaseous envelopes of the original binary, the precursor for double neutron stars proposed in the Brown (1995) scenario.

As the gravitational forces of the cores are softened at distances less than $\sim 0.1$, our dynamic simulations that lead to cores in a tight binary can provide only an upper limit on the separation at the end of their inspiral. Our simulations of the $m_{c}=0.15$ case, for example, place this upper limit at $\sim 0.03$ times the stellar radius (see Fig. 15). Thus, a binary composed of twin $M=10 M_{\odot}$, $R=200 R_{\odot}, M_{c}=1.5 M_{\odot}$ red giants would have their cores spiral to a separation of less than $0.03 R \approx 6 R_{\odot}$. The gradual transfer of energy to the circumbinary envelope could easily decrease the separation of the cores by a factor of $\sim 2$ further. As gravitational radiation alone can bring two $1.5 M_{\odot}$ point masses separated by up to $\sim 5 R_{\odot}$ to contact in less than a Hubble time, such systems could have evolved to become arbitrarily tight by the present time. We therefore believe that such double cores are indeed excellent candidates for binary NS progenitors, as proposed in the Brown scenario.

\subsection{Relevance to Planetary Nebulae}

Given the relatively short timescale covered by hydrodynamic simulations such as ours, the circumbinary en- 
Twin Binaries

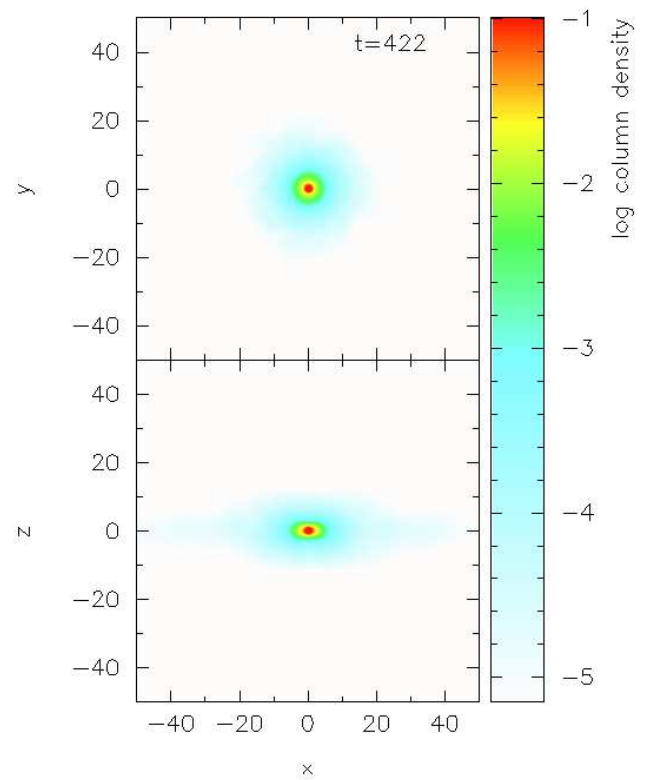

FIG. 13. - The state of the simulation presented in Fig. 12 at a late time $(t=422)$, with the column density projected in both the $x y$ and $x z$ planes.

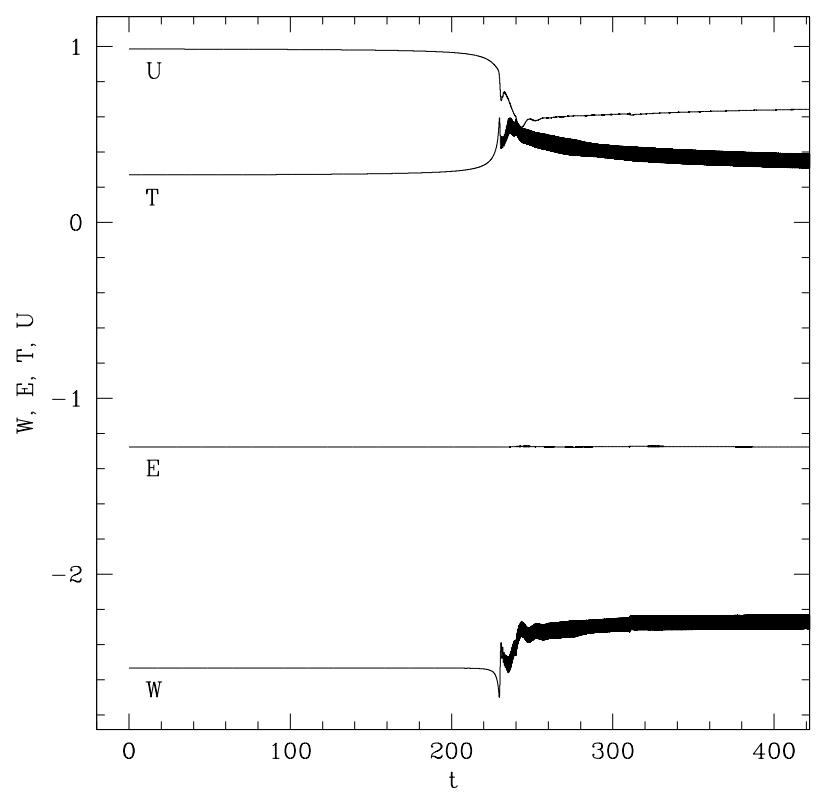

FIG. 14.- Energies versus time $t$ for the simulation presented in Fig. 12. From the bottom curve to the top one, we show gravitational energy $W$, total energy $E$, kinetic energy $T$, and internal energy $U$.

velopes at the end of our dynamical simulations are still optically thick. Nevertheless, it is natural to think of the final states of our merger calculations as a type of proto-PNe, specifically, as the immediate precursors of PNe with equal mass central binaries. Future work could use the end state of hydrodynamic calculations as initial conditions in models of PN evolution, with particular attention paid to any transition to the optically thin regime (revealing the central binary) and to the morphology of the gas distribution.
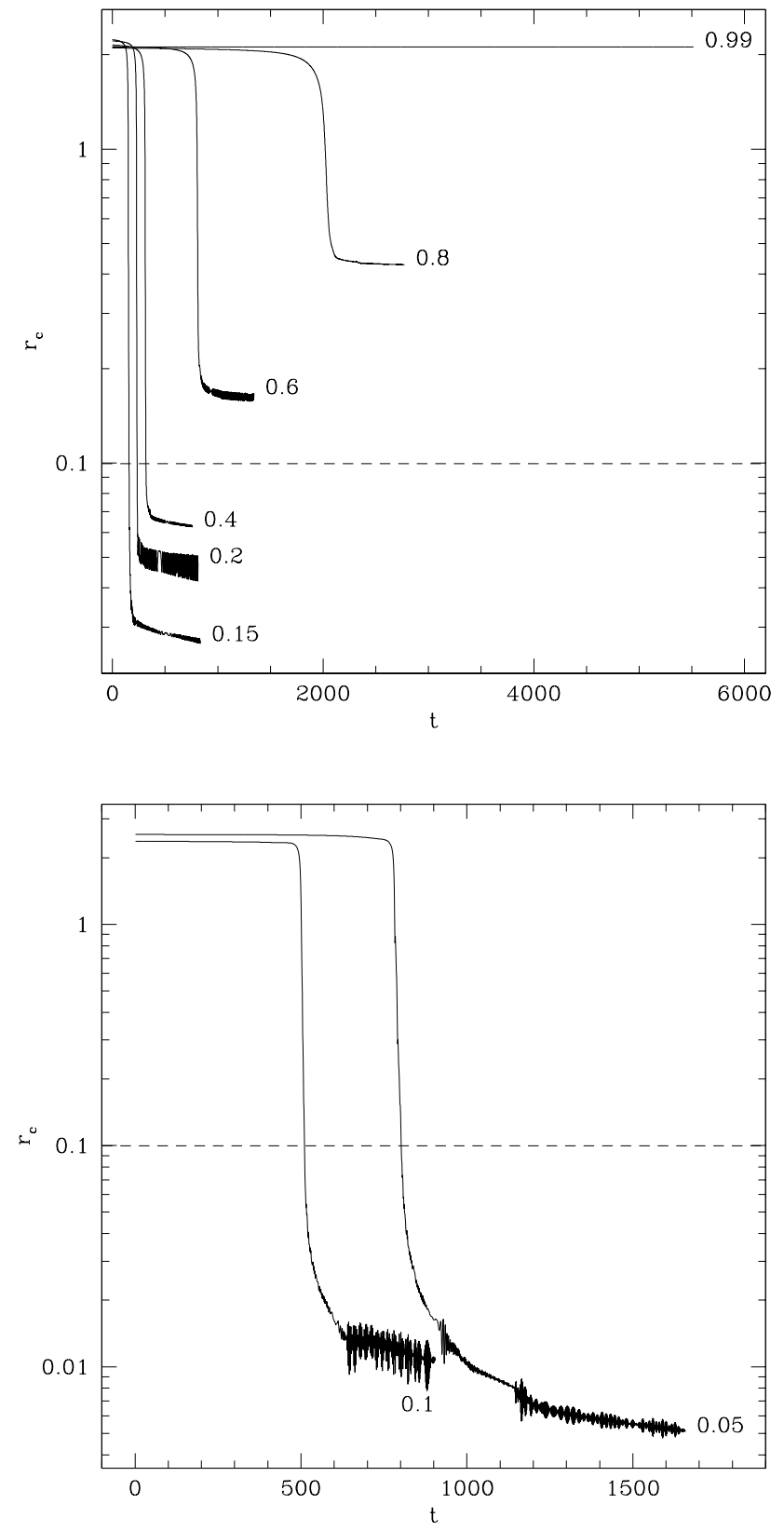

FIG. 15.- The separation $r_{c}$ between the cores as a function of time $t$ for binaries that begin just inside the Roche limit (top frame) and those that begin just inside the secular limit (bottom frame). Each curve is labeled by the factional core mass $m_{c}$. The horizontal dashed line indicates the minimum separation for which the gravitational interaction of the two cores is unsoftened in our simulations.

To examine whether the dynamical calculations of this paper yield final states that are consistent with the observations of the $q \approx 1$ double degenerate binaries, we consider a twin binary with $3 M_{\odot}$ components. We use the TWIN stellar evolution code to evolve each star in isolation. The calculation shows that the star expands to only $R \approx 30 R_{\odot}$ as it ascends the giant branch and to nearly $500 R_{\odot}$ on the asymptotic giant branch. Therefore, for any reasonable distribution of initial orbital separations, twin binaries made of such stars would much more often 


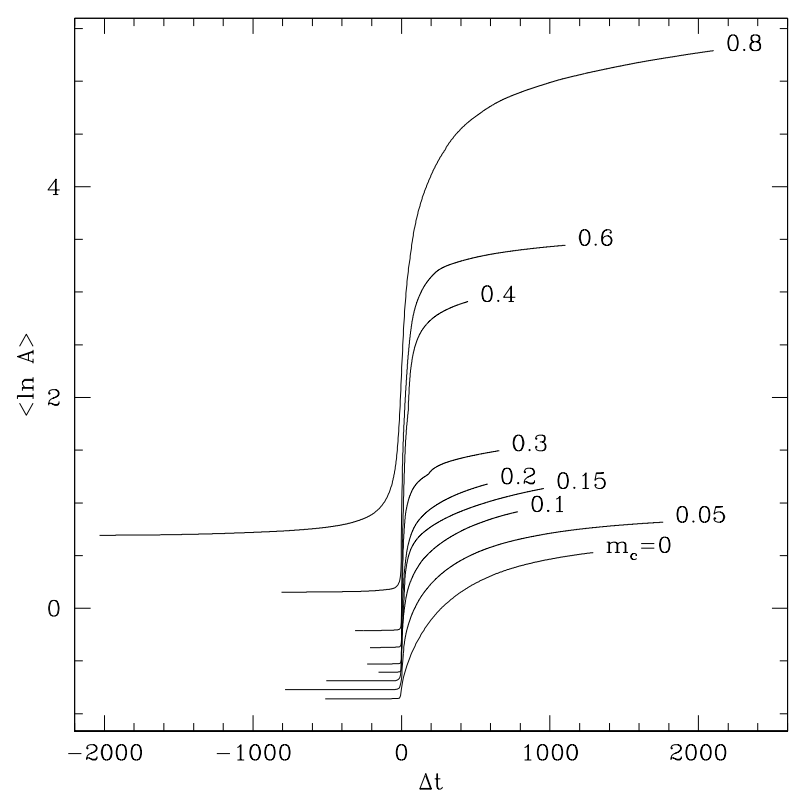

Fig. 16.- A measure of entropy evolution due to shock heating during several mergers. The natural logarithm of the entropic variable $A$ is averaged by mass over the gas of the system and plotted versus a shifted time coordinate $\Delta t$, chosen such that the inflection point in each curve (when the entropy is increasing most rapidly) occurs at $\Delta t=0$. Each curve is labeled by the factional core mass $m_{c}$ : as $m_{c}$ increases, so do both the initial and final values of $<\ln A>$. For each core mass, we present our simulation of largest initial separation that results in a merger, corresponding in Fig. 8 to the uppermost asterisk (for a given $m_{c}<0.15$ ) or filled square (for a given $m_{c} \geq 0.15$ ). Note that the initial value of the entropic variable $A$ equals the $K$ in equation (2), as calculated from the appropriate $E_{\mathrm{O}}$ in Table 1 . The entropic variable $A$ is in units of $G M^{1 / 3} R$.

come into contact while on the asymptotic giant branch than when on the red giant branch, consistent with the fact that most or all of the well-observed double degenerate stars in PNe seem to have masses too large to be He white dwarfs.

For the sake of discussion, consider such a twin binary that reaches the Roche limit when $R=200 R_{\odot}$. According to the stellar evolution calculation, an isolated star at that time would have a carbon-oxygen core of mass $M_{c}=0.57 M_{\odot}$ and, accounting for stellar winds, a total mass $M=2.8 M_{\odot}$. At this fractional core mass $m_{c}=0.57 / 2.8=0.20$, the dimensionless Roche separation (from the results of this paper) is $r_{\text {Roche }} \approx 2.2$, and thus the initial semimajor axis of this binary would have been $a=r_{\text {Roche }} R \approx 440 R_{\odot}$. From the $m_{c}=0.2$ curve in the top frame of Figure 15, we see we can place an upper limit of $\sim 0.05 R=10 R_{\odot}$ on the final separation of the two cores, which, from Kepler's third law, corresponds to an upper limit on the orbital period of 3 days. If instead $R=100 R_{\odot}$ at the time of merger, then a similar calculation gives a core mass $M_{c}=0.53 M_{\odot}$ and an upper limit on the orbital period of 0.9 days.

For comparison, the central binaries in NGC 6026, Abell 41, and Hen 2-428 have comparable masses to these core masses and orbital periods in roughly the 0.2 to 0.5 day range. We conclude that the results of our dynamical simulations, when applied to intermediate mass twin binaries, are consistent with observed characteristics of

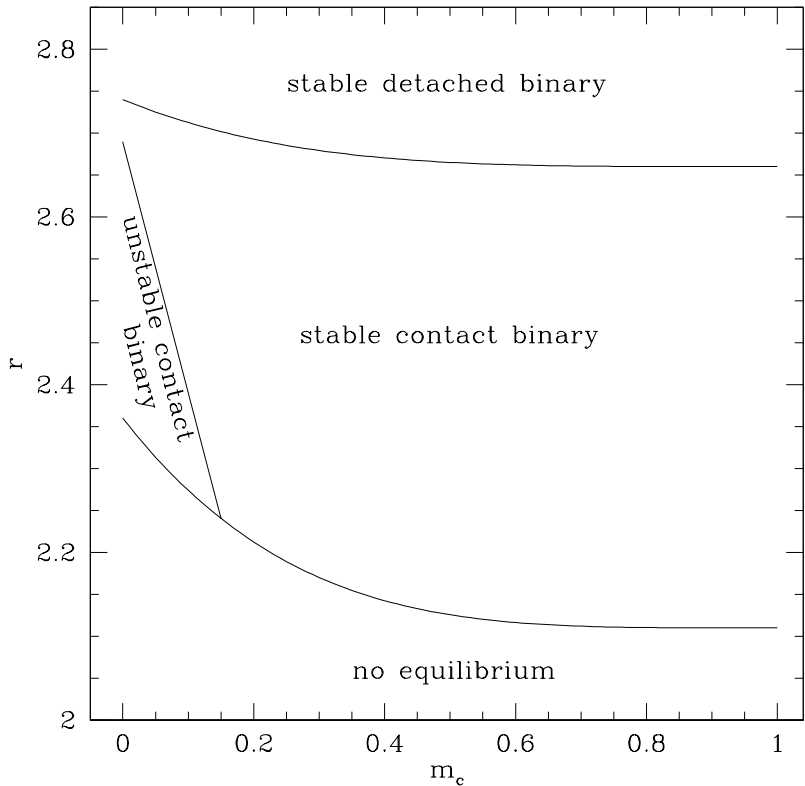

Fig. 17. - The parameter space of twin binaries. Here and throughout the paper, the core mass $m_{c}$ and binary separation $r$ are given in units of the total mass and radius, respectively, of an isolated binary component. The top curve, separating detached and contact binaries, marks configurations of first contact. The middle curve, spanning $0 \leq m_{c} \lesssim 0.15$, is the secular instability limit that separates stable and unstable contact binaries: in this work, we find that most secularly unstable systems are also dynamically unstable to mass transfer across the inner Lagrangian point. The bottom curve, separating contact binaries from configurations with no equilibrium, represents the Roche limit. Binaries that are unstable or that cannot exist in equilibrium have their components inspiral and merge, a process we follow with dynamical calculations.

double degenerate central binaries in PNe. In future simulations, a reduced gravitational softening between the two cores could allow for an even more precise determination of their final orbital separation. In this way, observed orbital parameters of degenerate binaries in $\mathrm{PNe}$ could be more readily connected to the properties of the parent stars from which they may have originated.

\subsection{Additional Comparisons and Future Work}

For core masses $m_{c} \gtrsim 0.15$, we find that a twin binary can exist stably in deep contact, at separations all the way down to the Roche limit. In contrast, the semi-analytic condensed polytrope models of Hjellming \& Webbink (1987) predict instead that twin binaries will experience sustained mass transfer once the components come in contact, provided only that $m_{c}<0.458$ (a range that includes the vast majority of giant stars). The primary oversimplification in the semi-analytic treatment appears to be the approximation that mass outside of the Roche lobe cannot help to contain the star within it. Our numerical calculations, however, model the common envelope that exists outside of the Roche lobe and that acts to suppress mass transfer. In addition, our fully three-dimensional calculations remove the point mass and spherical structure approximations implicit to the semi-analytic method.

The models of Hjellming \& Webbink (1987) seem best suited to semidetached binaries, where there is no com- 
mon envelope to complicate the dynamics of the mass flow. A comparison of such cases with our results is not possible, as our work is limited to binaries with identical components. Natural future work would include relaxing this constraint so that binaries with mass ratio $q \neq 1$ can be studied and compared with semi-analytic models. Of particular interest to the binary neutron star problem would be cases in which the mass ratio deviated from one by only a few percent or less.

The modeling of giants as $\Gamma=5 / 3$ condensed polytropes is a common simplifying approximation, one that here allows our results to be scaled to binaries of any mass and length scales. We note, however, that radiation pressure can be the dominant contributor to the equation of state at some ages and at some locations within the envelopes of massive giants $\left(M \gtrsim 14 M_{\odot}\right.$, according to calculations with the TWIN stellar evolution code). For such cases, our treatment of the envelope as a constant entropy, $\Gamma=5 / 3$ gas can be legitimately questioned. While the effects of employing more realistic stellar mod- els would be worthwhile to study in future work, we do not expect our results to change qualitatively. Regardless of the equation of state, gas that flows past the outer Lagrangian points will still necessarily carry away a specific angular momentum larger than the system average, forcing the remaining gas to configurations of smaller angular momentum per unit mass. We conclude that the inspiral of cores should be a common outcome whenever a real twin binary exceeds the Roche limit.

We thank Evghenii Gaburov, Zachary Proulx, Adam Simbeck, Eric Theriault, and the anonymous referee for helpful input. This material is based upon work supported by the National Science Foundation under grant no. 0703545 and has made use of both the SPLASH visualization software (Price 2007) and NASA's Astrophysics Data System. F.A.R. acknowledges support from NSF grant PHY-0855592. V.K. acknowledges support from NSF grant PHY-0969820.

\section{REFERENCES}

Athanassoula, E., Fady, E., Lambert, J. C., \& Bosma, A. 2000, MNRAS, 314,475

Belczynski, K., Kalogera, V., \& Bulik, T. 2002, ApJ, 572, 407

Burgay, M., et al. 2003, Nature, 426, 531

Bethe, H. A., \& Brown, G. E. 1998, ApJ, 506, 780

Bethe H. A., Brown G. E., Lee C.-H., 2007, PhR, 442, 5

Bhattacharya, D., \& van den Heuvel, E. P. J. 1991, Phys. Rep., 203, 1

Brown, G. E. 1995, ApJ, 440, 270

Bruch A. Vaz, L. P. R., \& Diaz, M. P. 2001, A\&A, 377, 898 B

Chandrasekhar, S. 1939, An introduction to the study of stellar structure (Chicago: The University of Chicago press)

Chandrasekhar, S. 1969, Ellipsoidal Figures of Equilibrium, New Haven: Yale University Press, 1969 (Revised Dover edition 1987)

Chandrasekhar, S. 1975, ApJ, 202, 809

Chevalier, R. A. 1993, ApJ, 411, L33

Counselman, C. C., III 1973, ApJ, 180, 307

Dan, M., Rosswog, S., \& Brüggen, M. 2009, Journal of Physics Conference Series, 172, 012034

De Marco, O. 2009, PASP, 121, 316

Dehnen, W. 2001, MNRAS, 324, 273

Dewi, J. D. M., \& van den Heuvel, E. P. J. 2004, MNRAS, 349 169

Dewi, J. D. M., Podsiadlowski, P., \& Sena, A. 2006, MNRAS, 368,1742

D'Souza, M. C. R., Motl, P. M., Tohline, J. E., \& Frank, J. 2006, ApJ, 643, 381

Eggleton P. P., 1971, MNRAS, 151, 351

Eggleton P. P., 1983, ApJ, 268, 368

Frank, J. 2008, New Astronomy Review, 51, 878

Fryer, C. L., Benz, W., \& Herant, M. 1996, ApJ, 460, 801

Gaburov, E., Lombardi, J., \& Portegies Zwart, S. 2010, MNRAS, 402,105

Geier, S., Napiwotzki, R., Heber, U., \& Nelemans, G. 2011, A\&A, 528, L16

Glebbeek E., Pols O. R., Hurley J. R., 2008, A\&A, 488, 1007

Habets, G. M. H. J. 1986, A\&A, 167, 61

Hachisu, I., \& Eriguchi, Y. 1984, PASJ, 36, 239

Härm, R., \& Schwarzschild, M. 1955, ApJS, 1, 319

Hernquist, L., \& Katz, N. 1989, ApJS, 70, 419

Hillwig, T. C. 2011, Asymmetric Planetary Nebulae 5 Conference

Hillwig, T. C., Bond, H. E., Afşar, M., \& De Marco, O. 2010, AJ 140,319

Hjellming, M. S., \& Webbink, R. F. 1987, ApJ, 318, 794

Hogeveen, S. J. 1992, Ap\&SS, 194, 143

Hogeveen, S. J. 1992, Ap\&SS, 195, 359

Hogeveen, S. J. 1992, Ap\&SS, 196, 299

Hulse, R. A., \& Taylor, J. H. 1975, ApJ, 195, L51

Hut, P. 1980, A\&A, 92, 167

Jacoby, B. A., Cameron, P. B., Jenet, F. A., Anderson, S. B., Murty, R. N., \& Kulkarni, S. R. 2006, ApJ, 644, L113

Jones, D., et al. 2010, MNRAS, 408, 2312

Kramer, M., et al. 2006, Science, 314, 97

Krumholz, M. R., \& Thompson, T. A. 2007, ApJ, 661, 1034

Lai, D., Rasio, F. A., \& Shapiro, S. L. 1993, ApJS, 88, 205
Lai, D., Rasio, F. A., \& Shapiro, S. L. 1993, ApJ, 406, L63 Lai, D., Rasio, F. A., \& Shapiro, S. L. 1994, ApJ, 420, 811 Lai, D., Rasio, F. A., \& Shapiro, S. L. 1994, ApJ, 423, 344 Lai, D., Rasio, F. A., \& Shapiro, S. L. 1994, ApJ, 437, 742 Lee C.-H., Park H.-J., Brown G. E., 2007, ApJ, 670, 741

Levine, A., Rappaport, S., Deeter, J. E., Boynton, P. E., \& Nagase, F. 1993, ApJ, 410, 328

Lombardi, J. C., Sills, A., Rasio, F. A., \& Shapiro, S. L. 1999, J. Comp. Phys., 152, 687

Lorén-Aguilar, P., Isern, J., \& García-Berro, E. 2009, A\&A, 500, 1193

Miszalski, B., Acker, A., Parker, Q. A., \& Moffat, A. F. J. 2009, A\&A, 505, 249

Motl, P. M., Tohline, J. E., \& Frank, J. 2002, ApJS, 138, 121

Nice, D. J., Sayer, R. W., \& Taylor, J. H. 1996, ApJ, 466, L87

Osterbrock, D. E. 1953, ApJ, 118, 529

Ostriker, J. P., \& Gunn, J. E. 1969, ApJ, 157, 1395

Paczyiński, B., \& Sienkiewicz, R. 1972, Acta Astronomica, 22, 73

Pinsonneault, M. H., \& Stanek, K. Z. 2006, ApJ, 639, L67

Piran, T., \& Shaviv, N. J. 2005, Physical Review Letters, 94, 051102

Pols, O. R. 1994, A\&A, 290, 119

Portegies Zwart S., et al., 2009, NewA, 14, 369

Price, D. J. 2007, Publ. Astron. Soc. Aust., 24, 159

Rasio, F. A. 1991, PhD Thesis, Cornell University

Rasio, F. A. 1994, Memorie della Societa Astronomica Italiana, 65,37

Rasio, F. A., \& Lombardi, J. C. 1999, J. Comp. App. Math., 109, 213

Rasio, F. A., \& Shapiro, S. L. 1991, ApJ, 377, 559

Rasio, F. A., \& Shapiro, S. L. 1992, ApJ, 401, 226

Rasio, F. A., \& Shapiro, S. L. 1994, ApJ, 432, 242

Rasio, F. A., \& Shapiro, S. L. 1995, ApJ, 438, 887

Rosswog S., 2009, NewAR, 53, 78

Rucinski, S. M. 1992, in The Realm of Interacting Binary Stars,

eds. J. Sahade et al. (Dordrecht: Kluwer), 177, 111

Santander-Garcia, M., Rodríguez-Gil, P., Jones, D., Corradi, R. L. M., Miszalski, B., Pyrzas, S., \& Rubio-Díez, M. M. 2011, Asymmetric Planetary Nebulae 5 Conference

Schönberg M., Chandrasekhar S., 1942, ApJ, 96, 161

Shimanskii, V. V., Borisov, N. V., Sakhibullin, N. A., \& Sheveleva, D. V. 2008, Astronomy Reports, 52, 479

Stairs, I. H., Thrsett, S. E., Taylor, J. H., \& Wolszczan, A. 2002 ApJ, 581, 501

Stairs, I. H., Thorsett, S. E., Dewey, R. J., Kramer, M., \& McPhee, C. A. 2006, MNRAS, 373, L50

Tassoul, M. 1975, ApJ, 202, 803

Thorsett, S. E., \& Chakrabarty, D. 1999, ApJ, 512, 288

Wang C., Lai D., Han J. L., 2006, ApJ, 639, 1007

Webbink, R. F. 1976, ApJ, 209, 829

Webbink, R. F. 2006, Journal of the American Association of Variable Star Observers (JAAVSO), 35, 124

Weisberg, J. M., \& Taylor, J. H. 2005, ASP Conf. Ser. 328:

Binary Radio Pulsars, 328, 25

Willems, B., \& Kalogera, V. 2004, ApJ, 603, L101

Willems, B., Kalogera, V., \& Henninger, M. 2004, ApJ, 616, 414

Wong, T.-W., Willems, B., \& Kalogera, V. 2010, ApJ, 721, 1689 